\title{
Topology of the gauge-invariant gauge field in two-color QCD.
}

\author{
Kurt Haller*, Lusheng Chen ${ }^{\dagger}$ and Y. S. Choi ${ }^{\ddagger}$ \\ University of Connecticut, Storrs, Connecticut 06269
}

\begin{abstract}
We investigate solutions to a nonlinear integral equation which has a central role in implementing the non-Abelian Gauss's Law and in constructing gaugeinvariant quark and gluon fields. Here we concern ourselves with solutions to this same equation that are not operator-valued, but are functions of spatial variables and carry spatial and $\mathrm{SU}(2)$ indices. We obtain an expression for the gauge-invariant gauge field in two-color QCD, define an index that we will refer to as the "winding number" that characterizes it, and show that this winding number is invariant to a small gauge transformation of the gauge field on which our construction of the gauge-invariant gauge field is based. We discuss the role of this gauge field in determining the winding number of the gauge-invariant gauge field. We also show that when the winding number of the gauge field is an integer $\ell \neq 0$, the gauge-invariant gauge field manifests winding numbers that are not integers, and are half-integers only when $\ell=0$.
\end{abstract}

\section{INTRODUCTION}

In earlier work, in which we discussed QCD in the temporal $\left(A_{i}^{\gamma}=0\right)$ gauge, [1] we implemented the non-Abelian Gauss's law and constructed quark and gluon operator-valued fields that are invariant to small non-Abelian gauge transformations. An essential element in that program was a nonlocal operator, $\overline{\mathcal{A}_{i}^{\gamma}}(\mathbf{r})$, which we called the "resolvent field" in later publications. [2,3] The resolvent field is a functional of the gauge field $A_{i}^{\gamma}(\mathbf{r})$, and it has a pivotal role in our work. It first appears, in combination with $\Pi_{i}^{\gamma}(\mathbf{r})$ - the canonical momentum for the gauge field and the negative chromoelectric field — in the operator,

$$
\Psi=\left\|\exp \left(i \int d \mathbf{r} \overline{\mathcal{A}}_{i}^{\gamma}(\mathbf{r}) \Pi_{i}^{\gamma}(\mathbf{r})\right)\right\|
$$

where the double-bar bracket denotes a normal ordering in which all the gauge fields are placed to the left of all canonical momenta, so that when the exponential is expanded,

\footnotetext{
*Department of Physics; e-mail: khaller@uconnvm.uconn.edu

$\dagger^{\dagger}$ Department of Physics; e-mail: chen@phys.uconn.edu

${ }^{\ddagger}$ Department of Mathematics; e-mail: choi@math.uconn.edu
} 
all powers of $\overline{\mathcal{A}}_{i}^{\gamma}(\mathbf{r})$ appear to the left of any of the $\Pi_{i}^{\gamma}(\mathbf{r})$. $\Psi$ converts any state $|\phi\rangle$ that obeys the Abelian Gauss's law $\partial_{i} \Pi_{i}^{\alpha}(\mathbf{r})|\phi\rangle=0$ into a state $\Psi|\phi\rangle$ that obeys the "pure-glue" non-Abelian Gauss's law $\left[\partial_{i} \Pi_{i}^{\alpha}(\mathbf{r})+g f^{\alpha \beta \gamma} A_{i}^{\beta}(\mathbf{r}) \Pi_{i}^{\gamma}(\mathbf{r})\right] \Psi|\phi\rangle=0$. 凹

The resolvent field reappears, in the form

$$
\overline{\mathcal{Y}^{\alpha}}(\mathbf{r})=\frac{\partial_{j}}{\partial^{2}} \overline{\mathcal{A}_{j}^{\alpha}}(\mathbf{r})
$$

in the expression for the gauge-invariant quark field

$$
\psi_{\mathrm{GI}}(\mathbf{r})=V_{\mathcal{C}}(\mathbf{r}) \psi(\mathbf{r})
$$

where

$$
V_{\mathcal{C}}(\mathbf{r})=\exp \left(-i g \overline{\mathcal{Y}^{\alpha}}(\mathbf{r}) \frac{\lambda^{\alpha}}{2}\right) \exp \left(-i g \mathcal{X}^{\alpha}(\mathbf{r}) \frac{\lambda^{\alpha}}{2}\right)
$$

and

$$
\mathcal{X}^{\alpha}(\mathbf{r})=\left[\frac{\partial_{i}}{\partial^{2}} A_{i}^{\alpha}(\mathbf{r})\right]
$$

The resolvent field also appears in the expression for the gauge-invariant gluon field

$$
\left[A_{\mathrm{Gl} i}^{\gamma}(\mathbf{r}) \frac{\lambda^{\gamma}}{2}\right]=V_{\mathcal{C}}(\mathbf{r})\left[A_{i}^{\gamma}(\mathbf{r}) \frac{\lambda^{\gamma}}{2}\right] V_{\mathcal{C}}^{-1}(\mathbf{r})+\frac{i}{g} V_{\mathcal{C}}(\mathbf{r}) \partial_{i} V_{\mathcal{C}}^{-1}(\mathbf{r})
$$

which can also be expressed as

$$
A_{\mathrm{Gl} i}^{\gamma}(\mathbf{r})=A_{T i}^{\gamma}(\mathbf{r})+\left[\delta_{i j}-\frac{\partial_{i} \partial_{j}}{\partial^{2}}\right] \overline{\mathcal{A}_{j}^{\gamma}}(\mathbf{r}) .
$$

In subsequent work, [2 [0] we used these gauge-invariant fields to derive an expression for a nonlocal interaction that couples gauge-invariant quark color-charge densities to each other and to gluons through "chains", in which each link consists of an integral over the gauge-invariant gluon field. $\mathrm{SU}(3)$ or $\mathrm{SU}(2)$ structure constants connect the links to form the chains. As was shown in Ref. [1], the quantities that appear in this formulation the gauge-invariant quark color-charge density $j_{0 \mathrm{Gl}}^{\alpha}=g \psi_{\mathrm{Gl}}^{\dagger} \frac{\lambda^{\alpha}}{2} \psi_{\mathrm{Gl}}$ and the gauge-invariant gauge field $A_{\mathrm{Gl} i}^{\gamma}(\mathbf{r})$ - commute with the generator of infinitesimal gauge transformations and therefore are completely invariant to small gauge transformations. In the work presented here, we will focus attention on the resolvent field in two-color QCD. We will examine its structure in configuration space and its role in determining the topological features of the gauge-invariant gluon field.

\footnotetext{
${ }^{1}$ We use non-relativistic notation in this work, in which subscripted indices designate contravariant components of contravariant quantities, such as $A_{i}^{\gamma}$, and covariant components of covariant quantities, such as $\partial_{i}$. This convention is used extensively throughout this work.
} 


\section{CONFIGURATIONS OF THE RESOLVENT FIELD}

In Ref. [1], we presented a nonlinear integral equation for the resolvent field. Although, in Ref. [1], our concern was with the series representation of the operator-valued resolvent field, it is also relevant to ask whether the integral equation for the resolvent field has solutions other than that series. It is this question that we now consider. The most general form of the integral equation for the resolvent field, applicable to any $\mathrm{SU}(\mathrm{N})$ gauge theory, is not useful for this investigation; but its $\mathrm{SU}(2)$ form, given in Ref. [1] , is very suitable for our purposes. In the $\mathrm{SU}(2)$ case, the integral equation for the resolvent field $\overline{\mathcal{A}_{i}^{\gamma}}(\mathbf{r})$ can be expressed in the following form:

$$
\overline{\mathcal{A}_{i}^{\gamma}}(\mathbf{r})=\overline{\mathcal{A}_{i}^{\gamma}}(\mathbf{r})_{\mathcal{X}}+\overline{\mathcal{A}_{i}^{\gamma}}(\mathbf{r})_{\overline{\mathcal{Y}}}
$$

where

$$
\begin{aligned}
\overline{\mathcal{A}_{i}^{\gamma}}(\mathbf{r})_{\mathcal{X}} & =g \epsilon^{\alpha \beta \gamma} \mathcal{X}^{\alpha}(\mathbf{r}) A_{i}^{\beta}(\mathbf{r}) \frac{\sin (\mathcal{N})}{\mathcal{N}} \\
& -g \epsilon^{\alpha \beta \gamma} \mathcal{X}^{\alpha}(\mathbf{r}) \partial_{i} \mathcal{X}^{\beta}(\mathbf{r}) \frac{1-\cos (\mathcal{N})}{\mathcal{N}^{2}} \\
& -g^{2} \epsilon^{\alpha \beta b} \epsilon^{b \mu \gamma} \mathcal{X}^{\mu}(\mathbf{r}) \mathcal{X}^{\alpha}(\mathbf{r}) A_{i}^{\beta}(\mathbf{r}) \frac{1-\cos (\mathcal{N})}{\mathcal{N}^{2}} \\
& +g^{2} \epsilon^{\alpha \beta b} \epsilon^{b \mu \gamma} \mathcal{X}^{\mu}(\mathbf{r}) \mathcal{X}^{\alpha}(\mathbf{r}) \partial_{i} \mathcal{X}^{\beta}(\mathbf{r})\left[\frac{1}{\mathcal{N}^{2}}-\frac{\sin (\mathcal{N})}{\mathcal{N}^{3}}\right]
\end{aligned}
$$

and

$$
\begin{aligned}
\overline{\mathcal{A}_{i}^{\gamma}}(\mathbf{r}) \overline{\mathcal{Y}} & =g \epsilon^{\alpha \beta \gamma} \overline{\mathcal{Y}^{\alpha}}(\mathbf{r})\left(A_{T i}^{\beta}(\mathbf{r})+\left(\delta_{i j}-\frac{\partial_{i} \partial_{j}}{\partial^{2}}\right) \overline{\mathcal{A}_{j}^{\beta}}(\mathbf{r})\right) \frac{\sin (\overline{\mathcal{N}})}{\overline{\mathcal{N}}} \\
& +g \epsilon^{\alpha \beta \gamma} \overline{\mathcal{Y}^{\alpha}}(\mathbf{r}) \partial_{i} \overline{\mathcal{Y}^{\beta}}(\mathbf{r}) \frac{1-\cos (\overline{\mathcal{N}})}{\overline{\mathcal{N}}^{2}} \\
& +g^{2} \epsilon^{\alpha \beta b} \epsilon^{b \mu \gamma} \overline{\mathcal{Y}^{\mu}}(\mathbf{r}) \overline{\mathcal{Y}^{\alpha}}(\mathbf{r})\left(A_{T i}^{\beta}(\mathbf{r})+\left(\delta_{i j}-\frac{\partial_{i} \partial_{j}}{\partial^{2}}\right) \overline{\mathcal{A}_{j}^{\beta}}(\mathbf{r})\right) \frac{1-\cos (\overline{\mathcal{N}})}{\overline{\mathcal{N}}^{2}} \\
& +g^{2} \epsilon^{\alpha \beta b} \epsilon^{b \mu \gamma} \overline{\mathcal{Y}^{\mu}}(\mathbf{r}) \overline{\mathcal{Y}^{\alpha}}(\mathbf{r}) \partial_{i} \overline{\mathcal{Y}^{\beta}}(\mathbf{r})\left[\frac{1}{\overline{\mathcal{N}}^{2}}-\frac{\sin (\overline{\mathcal{N}})}{\overline{\mathcal{N}}^{3}}\right] .
\end{aligned}
$$

We observe that the right-hand side of Eq. (2.2) is independent of $\overline{\mathcal{A}_{i}^{\gamma}}(\mathbf{r})$ and depends only on the gauge field $A_{i}^{\gamma}(\mathbf{r})$ and on trigonometric functions of $\mathcal{N}$, where

$$
\mathcal{N}=\left[g^{2} \mathcal{X}^{\alpha}(\mathbf{r}) \mathcal{X}^{\alpha}(\mathbf{r})\right]^{\frac{1}{2}}
$$

$\overline{\mathcal{A}_{i}^{\gamma}}(\mathbf{r})_{\mathcal{X}}$ therefore is not responsible for any of the nonlinearity of the nonlinear integral equation shown in Eq.(2.1), but represents an inhomogeneity in that equation. The right-hand side of Eq.(2.3), however, contains the resolvent field $\overline{\mathcal{A}_{i}^{\gamma}}(\mathbf{r})$ and $\overline{\mathcal{N}}$ as well as trigonometric functions of $\overline{\mathcal{N}}$, where

$$
\overline{\mathcal{N}}=\left[g^{2} \overline{\mathcal{Y}^{\alpha}}(\mathbf{r}) \overline{\mathcal{Y}^{\alpha}}(\mathbf{r})\right]^{\frac{1}{2}}
$$

$\overline{\mathcal{A}_{i}^{\gamma}}(\mathbf{r})_{\overline{\mathcal{Y}}}$ therefore is a nonlinear functional of the resolvent field $\overline{\mathcal{A}_{i}^{\gamma}}(\mathbf{r})$, and of $\overline{\mathcal{Y}^{\gamma}}(\mathbf{r})$, whose dependence on $\overline{\mathcal{A}_{i}^{\gamma}}(\mathbf{r})$ is given in Eq.(1.2). It is the structure of $\overline{\mathcal{A}_{i}^{\gamma}}(\mathbf{r}) \overline{\mathcal{Y}}$ that makes Eq.(2.1) a nonlinear integral equation. 
Eqs.(1.3) and (11.6) are identical in form to the equations describing the change in a spinor field and a gauge field, respectively, produced by a finite non-Abelian gauge transformation, with the important exception that the transforming $c$-number gauge function in a gauge transformation is replaced by the operator-valued $g \overline{\mathcal{Y}}^{\alpha}(\mathbf{r})$ and $g \mathcal{X}^{\alpha}(\mathbf{r})$ in $V_{\mathcal{C}}(\mathbf{r})$. The $\mathrm{SU}(2)$ version of $V_{\mathcal{C}}(\mathbf{r})$, given by

$$
\left[V_{\mathcal{C}}(\mathbf{r})\right]_{S U(2)}=\exp \left(-i g \overline{\mathcal{Y}^{\alpha}}(\mathbf{r}) \frac{\tau^{\alpha}}{2}\right) \exp \left(-i g \mathcal{X}^{\beta}(\mathbf{r}) \frac{\tau^{\beta}}{2}\right),
$$

can be interpreted as a sequence of two rotations represented in the fundamental representation of $\mathrm{SU}(2)$. In one of these rotations, $g \mathcal{X}^{\alpha}(\mathbf{r}) / \mathcal{N}$ designates a direction about which the rotation is made, and $\mathcal{N}$ the magnitude of the locally varying angle of rotation; a similar correspondence applies to $g \overline{\mathcal{Y}}^{\alpha}(\mathbf{r})$ and $\overline{\mathcal{N}}$ in the other rotation. In this $\mathrm{SU}(2)$ case, we can also express $\left[V_{\mathcal{C}}(\mathbf{r})\right]_{S U(2)}$ in the form

$$
\left[V_{\mathcal{C}}(\mathbf{r})\right]_{S U(2)}=\exp \left(-i g \overline{\mathcal{Z}^{\alpha}}(\mathbf{r}) \frac{\tau^{\alpha}}{2}\right)
$$

where the composition laws for rotations enable us to express $\overline{\mathcal{Z}^{\alpha}}(\mathbf{r})$ as a function of $\mathcal{X}^{\alpha}(\mathbf{r})$ and $\overline{\mathcal{Y}^{\alpha}}(\mathbf{r})$, and both in terms of equivalent sets of locally varying Euler angles, much as was done by Christ and Lee. [6] Because the algebra of $\mathrm{SU}(\mathrm{N})$ generators is closed, the $\mathrm{SU}(3)$ form of $V_{\mathcal{C}}(\mathbf{r})$ can also be expressed in the form $V_{\mathcal{C}}(\mathbf{r})=\exp \left(-i g \overline{\mathcal{Z}^{\alpha}}(\mathbf{r}) \frac{\lambda^{\alpha}}{2}\right)$, where the Baker-Hausdorff-Campbell theorem enables us to equate $\overline{\mathcal{Z}^{\alpha}}$ to a complicated expansion in $\mathcal{X}^{\alpha}$ and $\overline{\mathcal{Y}^{\alpha}}$. The familiar group composition laws for rotations are a special case of the Baker-Hausdorff-Campbell theorem applied to the $\mathrm{SU}(2)$ case. Eqs.(2.2) and (2.3) illustrate this similarity in form of Eqs.(1.3) and (1.6) to the equations that implement finite gauge transformations. Eq.(2.2) has the same form as a finite gauge transformation of a gauge field in the adjoint representation of $\mathrm{SU}(2)$, with $g \mathcal{X}^{\alpha}(\mathbf{r})$ serving as the function by which the field is gauge-transformed; the same applies to (2.3) with $g \overline{\mathcal{Y}}^{\alpha}(\mathbf{r})$ in place of $g \mathcal{X}^{\alpha}(\mathbf{r})$. Such nonAbelian gauge configurations have well-documented important geometric and topological implications for gauge theories. [0] 10] The similarity in form of the equations that establish gauge-invariant fields to those that effect gauge transformations accounts for the appearance in our work of expressions that resemble the inverse of the Faddeev Popov operator $\partial_{i}\left(\delta_{a c} \partial_{i}+\right.$ $g f^{c b a} A_{i}^{b}$ ), which arises when transformations from the temporal to the Coulomb gauge are carried out by treating them as transformations from Cartesian to curvilinear field variables. [6.11.12] In our work, $\partial_{i}\left(\delta_{a c} \partial_{i}+g f^{c b a} A_{\mathrm{Gl} i}^{b}\right)$ appears in place of the Faddeev-Popov operator, and it appears in a representation in which the gauge field $A_{\mathrm{Gl} i}^{b}$ and the quark color charge density $j_{0}^{b}=g \psi^{\dagger}\left(\lambda^{b} / 2\right) \psi$ are gauge invariant and in which the temporal gauge condition continues to apply, even though the gauge-invariant gauge field (but not the gauge field) is transverse. [4: [5]

In spite of this similarity in form of finite gauge transformations to Eqs.(1.3) and (1.6), the significance of these two equations is very different from that of a finite gauge transformation. In a gauge transformation, the gauge field is transformed by an arbitrary $c$-number function that bears no relationship to the original gauge field. But in the case of Eqs.(1.3) and (1.6), $\mathcal{X}^{\alpha}$ and $\overline{\mathcal{Y}^{\alpha}}$ (as well as $\mathcal{N}$ and $\overline{\mathcal{N}}$ ) are themselves functionals of the gauge field, and therefore are subject to the same gauge transformations that affect it. In fact, under the infinitesimal gauge transformation $\delta A_{i}^{\gamma}(\mathbf{r})=\partial_{i} \delta \omega^{\gamma}(\mathbf{r})+g \epsilon_{\gamma \alpha \beta} A_{i}^{\alpha}(\mathbf{r}) \delta \omega^{\beta}(\mathbf{r}), V_{\mathcal{C}}(\mathbf{r})$ transforms 
so that $\delta V_{\mathcal{C}}(\mathbf{r})=-i g V_{\mathcal{C}}(\mathbf{r}) \delta \omega^{\gamma}(\mathbf{r}) \frac{\lambda^{\gamma}}{2}$. [13] Instead of representing a gauge transformation, Eqs.(1.3) and (1.6) establish the operator-valued nonlocal quantities $\psi_{\mathrm{GI}}(\mathbf{r})$ and $A_{\mathrm{Gl} i}^{\gamma}(\mathbf{r})$, which are entirely invariant to any further transformations effected by the generator of gauge transformations

$$
\mathrm{T}_{G}=\exp \left[i \int d \mathbf{r}\left(\partial_{i} \Pi_{i}^{\gamma}(\mathbf{r})+g f^{\gamma \beta \alpha} A_{i}^{\beta}(\mathbf{r}) \Pi_{i}^{\alpha}(\mathbf{r})+g \psi^{\dagger}(\mathbf{r}) \frac{\lambda^{\gamma}}{2} \psi(\mathbf{r})\right) \omega^{\gamma}(\mathbf{r})\right] .
$$

The integral equation described in Eqs.(2.1)-(2.3) can be viewed in two distinct ways: On the one hand, as was shown in Ref. [1], Eqs.(2.1)-(2.3) can be used to generate an operatorvalued series representation of the resolvent field $\mathcal{A}_{i}^{\gamma}(\mathbf{r})$, which is instrumental in establishing gauge-invariant quark and gluon fields and quantum states that implement the non-Abelian Gauss's law. But, because all the operator-valued fields that appear in it commute with each other (the only operator that would not commute with the quantities in Eqs.(2.1)(2.3) - $\Pi_{i}^{\alpha}(\mathbf{r})$, the momentum conjugate to $A_{i}^{\alpha}(\mathbf{r})$ - does not appear in any of these equations), Eq.(2.1) also can be treated as a non-linear integral equation whose solutions can be investigated by more-or-less standard procedures. In this second context, we can view the resolvent field $\overline{\mathcal{A}_{i}^{\gamma}}(\mathbf{r})$ and the gauge field $A_{i}^{\gamma}(\mathbf{r})$ as functions of spatial variables that obey the same nonlinear integral equation as the corresponding operator-valued quantities. We can, furthermore, make an ansatz about the functional dependence of $\overline{\mathcal{A}_{i}^{\gamma}}(\mathbf{r})$ and $A_{i}^{\gamma}(\mathbf{r})$ on spatial variables that is physically reasonable, and that facilitates the solution of the nonlinear equation given in Eqs.(2.1)-(2.3). The functional forms of $\overline{\mathcal{A}_{i}^{\gamma}}(\mathbf{r})$ and $A_{i}^{\gamma}(\mathbf{r})$ will represent number-valued realizations of these operator-valued fields, and will enable us to investigate their spatial configurations and their topological features.

We will carry out this program by assuming that $\overline{\mathcal{A}_{i}^{\gamma}}(\mathbf{r})$ and $A_{i}^{\gamma}(\mathbf{r})$ are functions of spatial variables, that they are second-rank tensors in the combined spatial and $\mathrm{SU}(2)$ indices $i$ and $\gamma$ respectively, and that, except in so far as the forms of $\overline{\mathcal{A}_{i}^{\gamma}}(\mathbf{r})$ and $A_{i}^{\gamma}(\mathbf{r})$ must reflect this second-rank tensor structure, they are isotropic functions of the position. The most general form of $\overline{\mathcal{A}_{i}^{\gamma}}(\mathbf{r})$ will therefore be

$$
\overline{\mathcal{A}_{i}^{\gamma}}(\mathbf{r})=\delta_{i \gamma} \xi_{A}(r)+\frac{r_{i} r_{\gamma}}{r^{2}} \xi_{B}(r)+\epsilon_{i \gamma n} \frac{r_{n}}{r} \xi_{C}(r)
$$

where $\xi_{A}(r), \xi_{B}(r)$, and $\xi_{C}(r)$ represent as yet unspecified isotropic functions of $r$. It is convenient to separate the resolvent field $\overline{\mathcal{A}_{i}^{\gamma}}(\mathbf{r})$ into longitudinal and transverse parts, $\overline{\mathcal{A}_{i}^{\gamma}} L(\mathbf{r})$ and $\overline{\mathcal{A}}_{i}^{\gamma}{ }^{T}(\mathbf{r})$ respectively. We then use the representations

$$
{\overline{\mathcal{A}_{i}^{\gamma}}}^{L}(\mathbf{r})=\delta_{i \gamma} \Phi(r)+\frac{r_{i} r_{\gamma}}{r} \Phi^{\prime}(r) \quad \text { where } \Phi^{\prime}(r)=\frac{d \Phi}{d r}
$$

and

$$
\overline{\mathcal{A}}_{i}^{\gamma}(\mathbf{r})=\delta_{i \gamma} \varphi_{A}(r)+\frac{r_{i} r_{\gamma}}{r^{2}} \varphi_{B}(r)+\epsilon_{i \gamma n} \frac{r_{n}}{r} \varphi_{C}(r)
$$

where the transversality of $\overline{\mathcal{A}}_{i}^{\gamma}(\mathbf{r})$ requires that

$$
\frac{d\left(r^{2} \varphi_{B}\right)}{d r}+r^{2} \frac{d \varphi_{A}}{d r}=0 .
$$


The transversality of $\overline{\mathcal{A}_{i}^{\gamma}}{ }^{T}(\mathbf{r})$ does not impose any conditions on $\varphi_{C}$. On the basis of these considerations, we can write the resolvent field as

$$
\overline{\mathcal{A}_{i}^{\gamma}}(\mathbf{r})=\delta_{i \gamma}\left(\varphi_{A}+\Phi\right)+\frac{r_{i} r_{\gamma}}{r^{2}}\left(\varphi_{B}+r \Phi^{\prime}\right)+\epsilon_{i \gamma n} \frac{r_{n}}{r} \varphi_{C},
$$

and $\overline{\mathcal{Y}^{\alpha}}(\mathbf{r})$ becomes

$$
\overline{\mathcal{Y}^{\alpha}}(\mathbf{r})=r_{\alpha} \Phi(r)
$$

The same analysis can be applied to the gauge field $A_{i}^{\gamma}(\mathbf{r})$, with the result that we obtain the wholly analogous equations

$$
A_{i}^{\gamma L}(\mathbf{r})=\delta_{i \gamma} \mathcal{S}(r)+\frac{r_{i} r_{\gamma}}{r} \mathcal{S}^{\prime}(r)
$$

and

$$
A_{i}^{\gamma T}(\mathbf{r})=\delta_{i \gamma} \mathcal{T}_{A}(r)+\frac{r_{i} r_{\gamma}}{r^{2}} \mathcal{T}_{B}(r)+\epsilon_{i \gamma n} \frac{r_{n}}{r} \mathcal{T}_{C}(r)
$$

with

$$
\frac{d\left(r^{2} \mathcal{T}_{B}\right)}{d r}+r^{2} \frac{d \mathcal{T}_{A}}{d r}=0
$$

The complete gauge field can be represented as

$$
A_{i}^{\gamma}(\mathbf{r})=\frac{1}{g r}\left\{\left(\delta_{i \gamma}-\frac{r_{i} r_{\gamma}}{r^{2}}\right) \mathcal{N}+\frac{r_{i} r_{\gamma}}{r} \frac{d \mathcal{N}}{d r}\right\}+\delta_{i \gamma} \mathcal{T}_{A}+\frac{r_{i} r_{\gamma}}{r^{2}} \mathcal{T}_{B}+\epsilon_{i \gamma n} \frac{r_{n}}{r} \mathcal{T}_{C},
$$

where we have used Eqs.2.4) and (2.15) to set $\operatorname{gr} \mathcal{S}=\mathcal{N}$.

We can use the representations given in Eqs.(2.10), (2.11), (2.15), and (2.16) to express Eq.(2.3) as

$$
\begin{aligned}
\overline{\mathcal{A}_{i}^{\gamma}} \overline{\mathcal{Y}} & =\left\{\epsilon_{i \gamma n} \frac{r_{n}}{r}\left[\varphi_{A}+\mathcal{T}_{A}\right]-\left(\delta_{i \gamma}-\frac{r_{i} r_{\gamma}}{r^{2}}\right)\left[\varphi_{C}+\mathcal{T}_{C}\right]\right\} \sin \overline{\mathcal{N}} \\
& +\frac{1}{g r} \epsilon_{i \gamma n} \frac{r_{n}}{r}(1-\cos \overline{\mathcal{N}}) \\
& +\left\{\left(\delta_{i \gamma}-\frac{r_{i} r_{\gamma}}{r^{2}}\right)\left[\varphi_{A}+\mathcal{T}_{A}\right]+\epsilon_{i \gamma n} \frac{r_{n}}{r}\left[\varphi_{C}+\mathcal{T}_{C}\right]\right\}(1-\cos \overline{\mathcal{N}}) \\
& +\frac{1}{g r}\left(\delta_{i \gamma}-\frac{r_{i} r_{\gamma}}{r^{2}}\right)(\overline{\mathcal{N}}-\sin \overline{\mathcal{N}})
\end{aligned}
$$

where we have used Eqs.(2.5) and (2.14) to set $\operatorname{gr} \Phi=\overline{\mathcal{N}}$. In a similar way, we can express Eq.(2.2) as

$$
\begin{aligned}
\overline{\mathcal{A}_{i}^{\gamma}} \overline{\mathcal{X}} & =\left\{\epsilon_{i \gamma n} \frac{r_{n}}{r}\left[\frac{\mathcal{N}}{g r}+\mathcal{T}_{A}\right]-\left(\delta_{i \gamma}-\frac{r_{i} r_{\gamma}}{r^{2}}\right) \mathcal{T}_{C}\right\} \sin \mathcal{N} \\
& -\frac{1}{g r} \epsilon_{i \gamma n} \frac{r_{n}}{r}(1-\cos \mathcal{N}) \\
& -\left\{\left(\delta_{i \gamma}-\frac{r_{i} r_{\gamma}}{r^{2}}\right)\left[\frac{\mathcal{N}}{g r}+\mathcal{T}_{A}\right]+\epsilon_{i \gamma n} \frac{r_{n}}{r} \mathcal{T}_{C}\right\}(1-\cos \mathcal{N}) \\
& +\frac{1}{g r}\left(\delta_{i \gamma}-\frac{r_{i} r_{\gamma}}{r^{2}}\right)(\mathcal{N}-\sin \mathcal{N}) .
\end{aligned}
$$


It is manifest from Eqs. $(2.19)$ and $(2.20)$ that $r_{\gamma} \overline{\mathcal{A}_{i}^{\gamma}}(\mathbf{r}) \overline{\mathcal{X}}=0$ and $r_{\gamma} \overline{\mathcal{A}_{i}^{\gamma}}(\mathbf{r})_{\overline{\mathcal{Y}}}=0$, so that $r_{\gamma} \overline{\mathcal{A}_{i}^{\gamma}}(\mathbf{r})=0$ too. When we use that fact in Eq. 2.13$)$, we find that

$$
\Phi+r \Phi^{\prime}+\varphi_{A}+\varphi_{B}=0 \quad \text { or, equivalently, } \quad \frac{\overline{\mathcal{N}}^{\prime}}{g}+\varphi_{A}+\varphi_{B}=0
$$

enabling us to represent $\overline{\mathcal{A}_{i}^{\gamma}}(\mathbf{r})$ as

$$
\overline{\mathcal{A}_{i}^{\gamma}}(\mathbf{r})=\left(\delta_{i \gamma}-\frac{r_{i} r_{\gamma}}{r^{2}}\right)\left(\frac{\overline{\mathcal{N}}}{g r}+\varphi_{A}\right)+\epsilon_{i \gamma n} \frac{r_{n}}{r} \varphi_{C} .
$$

Substitution of Eqs.(2.19), 2.20), and (2.22) into Eq.(2.1) enables us to express $\varphi_{A}$ and $\varphi_{C}$ as functions of $\overline{\mathcal{N}}, \mathcal{N}, \mathcal{T}_{A}$, and $\mathcal{T}_{C}$, so that we obtain

$$
\varphi_{A}=\frac{1}{g r}[\mathcal{N} \cos (\overline{\mathcal{N}}+\mathcal{N})-\sin (\overline{\mathcal{N}}+\mathcal{N})]+\mathcal{T}_{A}[\cos (\overline{\mathcal{N}}+\mathcal{N})-1]-\mathcal{T}_{C} \sin (\overline{\mathcal{N}}+\mathcal{N})
$$

and

$$
\varphi_{C}=\frac{1}{g r}[\mathcal{N} \sin (\overline{\mathcal{N}}+\mathcal{N})+\cos (\overline{\mathcal{N}}+\mathcal{N})-1]+\mathcal{T}_{C}[\cos (\overline{\mathcal{N}}+\mathcal{N})-1]+\mathcal{T}_{A} \sin (\overline{\mathcal{N}}+\mathcal{N})
$$

We can solve Eqs.(2.12) and (2.21) simultaneously to eliminate $\varphi_{B}$, and obtain a nonlinear differential equation that is equivalent to the nonlinear integral equation described in Eqs.(2.1)-(2.3) modulo the previously-mentioned assumptions about the forms of the resolvent field and the gauge field. It is most convenient to use the dimensionless variable $u=\ln \left(r / r_{0}\right)$, where $r_{0}$ is an arbitrary constant length, as the independent variable in expressing this differential equation, which then is

$$
\begin{aligned}
\frac{d^{2} \overline{\mathcal{N}}}{d u^{2}} & +\frac{d \overline{\mathcal{N}}}{d u}+2[\mathcal{N} \cos (\overline{\mathcal{N}}+\mathcal{N})-\sin (\overline{\mathcal{N}}+\mathcal{N})] \\
& +2 g r_{0} \exp (u)\left\{\mathcal{T}_{A}[\cos (\overline{\mathcal{N}}+\mathcal{N})-1]-\mathcal{T}_{C} \sin (\overline{\mathcal{N}}+\mathcal{N})\right\}=0 .
\end{aligned}
$$

Eq.(2.25) is a nonlinear differential equation in the variable $\overline{\mathcal{N}}$, which determines the resolvent field. $\mathcal{N}, \mathcal{T}_{A}$, and $\mathcal{T}_{C}$ will be taken to be known functions in this equation. One question of immediate interest is whether there are nonvanishing solutions for the case that $\mathcal{N}, \mathcal{T}_{A}$, and $\mathcal{T}_{C}$ all vanish, so that the gauge field $A_{i}^{\gamma}$ is identically zero. In that case, Eq.(2.25) reduces to

$$
\frac{d^{2} \overline{\mathcal{N}}}{d u^{2}}+\frac{d \overline{\mathcal{N}}}{d u}-2 \sin (\overline{\mathcal{N}})=0
$$

We observe that Eq. (2.26) is the equation for a damped pendulum in which small-amplitude oscillations have not been assumed, in which $u$ corresponds to the time, and $\overline{\mathcal{N}}=\theta+\pi$, where $\theta=0$ when the pendulum is in its stable equilibrium position. Moreover, Eq.(2.26) corresponds to the equation for the pendulum in which mass, length, local acceleration due to gravity, and damping constant have been set $=1$, so that no adjustable parameters remain in the equation. 
In discussing Eqs.(2.25) and (2.26), we will assume that $\mathcal{T}_{A}, \mathcal{T}_{C}$, and $\mathcal{N}-2 \ell \pi$ (where $\ell$ is an integer), vanish faster than $1 / r$ as $r \rightarrow \infty$. Moreover, we also assume that the gauge field is bounded everywhere, so that $r \mathcal{T}_{A} \rightarrow 0$ as $r \rightarrow 0$, and that the same applies to $r \mathcal{T}_{C}$, and $r \mathcal{N}$. We will make these conditions more precise in the Appendix.

The fact that Eq.(2.26) describes a damped pendulum moving without a driving force makes it obvious that it has nonvanishing solutions. In the limit $u \rightarrow \infty$, the damped pendulum must come to rest in a position of static equilibrium, so that, as $u \rightarrow \infty, \overline{\mathcal{N}} \rightarrow m \pi$, where $m$ is an arbitrary integer. Physical considerations lead us to expect that the damped pendulum will come to rest in a position of stable equilibrium, for which $\overline{\mathcal{N}} \rightarrow(2 m+1) \pi$. But in the application of Eq.(2.26) of interest to us in the current context, all possible solutions - those that terminate at saddle points as well as those that terminate at stable equilibrium positions - must be considered as long as they are bounded functions in the interval $-\infty<u<\infty$. Eq. (2.25) is more complicated than Eq.(2.26); but as a result of the conditions on $\mathcal{T}_{A}$, and $\mathcal{T}_{C}$, and on $\mathcal{N}$ for the $\ell=0$ case, discussed above and made precise in the Appendix, Eq. (2.25) approaches Eq.(2.26) at the origin in configuration space, i.e. in the limit $u \rightarrow-\infty$, as well as in the limit $u \rightarrow \infty$. It is therefore reasonable to expect that $\overline{\mathcal{N}} \rightarrow m \pi$ as $u \rightarrow \infty$ even when it is governed by Eq.(2.25). That this expectation is fulfilled, is shown in the Appendix. When $\ell \neq 0, \overline{\mathcal{N}}$ still approaches a definite limit as $u \rightarrow \infty$, but that limit is no longer an integer multiple of $\pi$. This case is discussed in Section [II, and the mathematical foundations for this discussion are given in the Appendix.

It is possible to use the results obtained in this section, in particular Eqs.(2.11), (2.16), (2.23) and (2.24), to represent the gauge-invariant gauge field as

$$
\begin{aligned}
& A_{\mathrm{G} \mid i}^{\gamma}(\mathbf{r})=\frac{1}{g r}\left\{\epsilon_{i \gamma n} \frac{r_{n}}{r}[\cos (\overline{\mathcal{N}}+\mathcal{N})-1+\mathcal{N} \sin (\overline{\mathcal{N}}+\mathcal{N})]+\left(\delta_{i \gamma}-\frac{r_{i} r_{\gamma}}{r^{2}}\right) \times\right. \\
& \left.\times[\mathcal{N} \cos (\overline{\mathcal{N}}+\mathcal{N})-\sin (\overline{\mathcal{N}}+\mathcal{N})]-\frac{r_{i} r_{\gamma}}{r} \frac{d \overline{\mathcal{N}}}{d r}\right\} \\
& +\mathcal{T}_{A}\left\{\left(\delta_{i \gamma}-\frac{r_{i} r_{\gamma}}{r^{2}}\right) \cos (\overline{\mathcal{N}}+\mathcal{N})+\epsilon_{i \gamma n} \frac{r_{n}}{r} \sin (\overline{\mathcal{N}}+\mathcal{N})\right\}+\frac{r_{i} r_{\gamma}}{r^{2}}\left(\mathcal{T}_{A}+\mathcal{T}_{B}\right) \\
& +\mathcal{T}_{C}\left\{\epsilon_{i \gamma n} \frac{r_{n}}{r} \cos (\overline{\mathcal{N}}+\mathcal{N})-\left(\delta_{i \gamma}-\frac{r_{i} r_{\gamma}}{r^{2}}\right) \sin (\overline{\mathcal{N}}+\mathcal{N})\right\} .
\end{aligned}
$$

When we set the entire gauge field $A_{i}^{\gamma}=0$, we obtain

$$
\left[A_{\mathrm{Gl} i}^{\gamma}(\mathbf{r})\right]_{\left(A_{i}^{\gamma}=0\right)}=\frac{1}{g r}\left\{\epsilon_{i \gamma n} \frac{r_{n}}{r}[\cos (\overline{\mathcal{N}})-1]-\left(\delta_{i \gamma}-\frac{r_{i} r_{\gamma}}{r^{2}}\right) \sin \overline{\mathcal{N}}-\frac{r_{i} r_{\gamma}}{r} \frac{d \overline{\mathcal{N}}}{d r}\right\} .
$$

As a consequence of Eq.(2.25), $\partial_{i} A_{\mathrm{Gl} i}^{\gamma}(\mathbf{r})=0$, and similarly, $\partial_{i}\left[A_{\mathrm{Gl} i}^{\gamma}(\mathbf{r})\right]_{\left(A_{i}^{\gamma}=0\right)}=0$ follows from Eq.(2.26), so that the consistency of Eqs.(2.27) and (2.28) with the transversality of the gauge-invariant gauge field is confirmed.

In order to investigate the changes in $\overline{\mathcal{N}}$ that can ensue from gauge transformations, we turn our attention to the changes $\delta \mathcal{N}, \delta \mathcal{T}_{A}, \delta \mathcal{T}_{B}$, and $\delta \mathcal{T}_{C}$ in the corresponding quantities $\mathcal{N}, \mathcal{T}_{A}, \mathcal{T}_{B}$, and $\mathcal{T}_{C}$ due to the infinitesimal $\mathrm{SU}(2)$ gauge transformation

$$
\delta A_{i}^{\gamma}(\mathbf{r})=\partial_{i} \delta \omega^{\gamma}(\mathbf{r})+g \epsilon_{\gamma \alpha \beta} A_{i}^{\alpha}(\mathbf{r}) \delta \omega^{\beta}(\mathbf{r}) .
$$


We extend the ansatz that led to Eqs.(2.15) and (2.16) to choose an expression for $\delta \omega^{\gamma}(\mathbf{r})$ that is isotropic except in so far as it transforms as a vector in the adjoint representation of $\mathrm{SU}(2)$. We therefore represent $\delta \omega^{\gamma}(\mathbf{r})$ as

$$
\delta \omega^{\gamma}(\mathbf{r})=\frac{r_{\gamma}}{r} \Delta(r)
$$

where $\Delta(r)$ is an infinitesimal, isotropic quantity. We substitute $\delta \mathcal{N}, \delta \mathcal{T}_{A}, \delta \mathcal{T}_{B}$, and $\delta \mathcal{T}_{C}$ for $\mathcal{N}, \mathcal{T}_{A}, \mathcal{T}_{B}$, and $\mathcal{T}_{C}$ respectively, in Eq.(2.18), to represent $\delta A_{i}^{\gamma}(\mathbf{r})$, the infinitesimal change in $A_{i}^{\gamma}(\mathbf{r})$ due to a gauge transformation, and obtain

$$
\delta A_{i}^{\gamma}(\mathbf{r})=\left(\delta_{i \gamma}-\frac{r_{i} r_{\gamma}}{r^{2}}\right) \frac{\delta \mathcal{N}}{g r}+\frac{r_{i} r_{\gamma}}{g r^{2}} \frac{d(\delta \mathcal{N})}{d r}+\delta_{i \gamma} \delta \mathcal{T}_{A}(r)+\frac{r_{i} r_{\gamma}}{r^{2}} \delta \mathcal{T}_{B}(r)+\epsilon_{i \gamma n} \frac{r_{n}}{r} \delta \mathcal{T}_{C}(r) .
$$

We then substitute Eq.(2.31) in the left-hand side of Eq. (2.29), and Eq.(2.18) in the righthand side to obtain

$$
\begin{gathered}
\frac{\delta \mathcal{N}(r)}{g r}+\delta \mathcal{T}_{A}(r)=\frac{\Delta(r)}{r}+g \Delta(r) \mathcal{T}_{C}(r), \\
r \frac{d}{d r}\left(\frac{\delta \mathcal{N}(r)}{g r}\right)+\delta \mathcal{T}_{B}(r)=-\frac{\Delta(r)}{r}+\frac{d}{d r} \Delta(r)-g \Delta(r) \mathcal{T}_{C}(r),
\end{gathered}
$$

and

$$
\delta \mathcal{T}_{C}(r)=-g \Delta(r)\left(\frac{\mathcal{N}(r)}{g r}+\mathcal{T}_{A}(r)\right) .
$$

We combine Eqs.(2.17), (2.32) and (2.33) to obtain

$$
r^{2} \frac{d^{2}}{d r^{2}} \delta \phi(r)+2 r \frac{d}{d r} \delta \phi(r)-2 \delta \phi(r)=-2 g r \Delta(r) T_{C}(r)
$$

where we have set

$$
\frac{\delta \mathcal{N}(r)}{g}-\Delta(r)=\delta \phi(r)
$$

With a little more algebra we can also obtain

$$
\delta \mathcal{T}_{A}(r)=-\frac{r}{2} \frac{d^{2}}{d r^{2}} \delta \phi(r)-\frac{d}{d r} \delta \phi(r)
$$

and

$$
\delta \mathcal{T}_{B}(r)=\frac{r}{2} \frac{d^{2}}{d r^{2}} \delta \phi(r)
$$

To investigate the changes that gauge transformations induce in $\overline{\mathcal{N}}$, we examine Eqs.(2.23) and (2.24) to find relationships between the changes that infinitesimal gauge transformations produce in the quantities that appear in these two equations. We find that 


$$
\begin{aligned}
\delta \varphi_{A} & =\frac{1}{g r}\{\delta \mathcal{N} \cos (\overline{\mathcal{N}}+\mathcal{N})-[\mathcal{N} \sin (\overline{\mathcal{N}}+\mathcal{N})+\cos (\overline{\mathcal{N}}+\mathcal{N})](\delta \overline{\mathcal{N}}+\delta \mathcal{N})\} \\
& +\delta \mathcal{T}_{A}[\cos (\overline{\mathcal{N}}+\mathcal{N})-1]-\mathcal{T}_{A} \sin (\overline{\mathcal{N}}+\mathcal{N})(\delta \overline{\mathcal{N}}+\delta \mathcal{N}) \\
& -\delta \mathcal{T}_{C} \sin (\overline{\mathcal{N}}+\mathcal{N})-\mathcal{T}_{C} \cos (\overline{\mathcal{N}}+\mathcal{N})(\delta \overline{\mathcal{N}}+\delta \mathcal{N})
\end{aligned}
$$

and

$$
\begin{aligned}
\delta \varphi_{C} & =\frac{1}{g r}\{\delta \mathcal{N} \sin (\overline{\mathcal{N}}+\mathcal{N})+[\mathcal{N} \cos (\overline{\mathcal{N}}+\mathcal{N})-\sin (\overline{\mathcal{N}}+\mathcal{N})](\delta \overline{\mathcal{N}}+\delta \mathcal{N})\} \\
& +\delta \mathcal{T}_{C}[\cos (\overline{\mathcal{N}}+\mathcal{N})-1]-\mathcal{T}_{C} \sin (\overline{\mathcal{N}}+\mathcal{N})(\delta \overline{\mathcal{N}}+\delta \mathcal{N}) \\
& +\delta \mathcal{T}_{A} \sin (\overline{\mathcal{N}}+\mathcal{N})+\mathcal{T}_{A} \cos (\overline{\mathcal{N}}+\mathcal{N})(\delta \overline{\mathcal{N}}+\delta \mathcal{N})
\end{aligned}
$$

As shown in Eq.(1.7), changes produced by gauge transformations in the transverse components of $A_{i}^{\gamma}$ and $\overline{\mathcal{A}}_{i}^{\gamma}$ cancel, so that

$$
\delta \varphi_{A}+\delta \mathcal{T}_{A}=0, \quad \delta \varphi_{B}+\delta \mathcal{T}_{B}=0, \text { and } \delta \varphi_{C}+\delta \mathcal{T}_{C}=0
$$

We substitute Eq.(2.41) in Eqs.(2.39) and (2.40), set

$$
X=\mathcal{T}_{C}+\frac{1}{g r}, \quad Y=\mathcal{T}_{A}+\frac{\mathcal{N}}{g r}, \theta=\overline{\mathcal{N}}+\mathcal{N}, \text { and } \xi=\delta \overline{\mathcal{N}}+\delta \mathcal{N}-g \Delta,
$$

and find that

$$
(X \cos \theta+Y \sin \theta) \xi=0
$$

and

$$
(X \sin \theta-Y \cos \theta) \xi=0 .
$$

Since $X$ and $Y$ are determined by the initial configuration of the gauge field $A_{i}^{\gamma}$, which is arbitrary, Eqs.(2.43) and (2.44) must be satisfied without requiring that $X$ and $Y$ vanish. It therefore follows that $\xi=0$, which, with Eq.(2.36), leads to

$$
\delta \overline{\mathcal{N}}=-g \delta \phi
$$

and, with Eq.(2.35), to the following differential equation for the variation of $\overline{\mathcal{N}}$ produced by an infinitesimal gauge transformation:

$$
r^{2} \frac{d^{2}}{d r^{2}} \delta \overline{\mathcal{N}}(r)+2 r \frac{d}{d r} \delta \overline{\mathcal{N}}(r)-2 \delta \overline{\mathcal{N}}(r)=2 g^{2} \Delta(r) r \mathcal{T}_{C}(r) .
$$

Further confirmation of the consistency of these results is obtained by examining the variation of $A_{\mathrm{Gl} i}^{\gamma}(\mathbf{r})$ due to an infinitesimal gauge transformation of the quantities that constitute it. This variation is given by 


$$
\begin{aligned}
& \delta A_{\mathrm{Gl} i}^{\gamma}(\mathbf{r})=\frac{1}{g r}\left\{\epsilon_{i \gamma n} \frac{r_{n}}{r}[\delta \mathcal{N} \sin (\overline{\mathcal{N}}+\mathcal{N})+(\mathcal{N} \cos (\overline{\mathcal{N}}+\mathcal{N})-\sin (\overline{\mathcal{N}}+\mathcal{N})) \delta(\overline{\mathcal{N}}+\mathcal{N})]+\right. \\
& \left.\left(\delta_{i \gamma}-\frac{r_{i} r_{\gamma}}{r^{2}}\right)[\delta \mathcal{N} \cos (\overline{\mathcal{N}}+\mathcal{N})-(\mathcal{N} \sin (\overline{\mathcal{N}}+\mathcal{N})+\cos (\overline{\mathcal{N}}+\mathcal{N})) \delta(\overline{\mathcal{N}}+\mathcal{N})]-\frac{r_{i} r_{\gamma}}{r} \frac{d(\delta \overline{\mathcal{N}})}{d r}\right\} \\
& +\delta \mathcal{T}_{A}\left\{\left(\delta_{i \gamma}-\frac{r_{i} r_{\gamma}}{r^{2}}\right) \cos (\overline{\mathcal{N}}+\mathcal{N})+\epsilon_{i \gamma n} \frac{r_{n}}{r} \sin (\overline{\mathcal{N}}+\mathcal{N})\right\}+\frac{r_{i} r_{\gamma}}{r^{2}}\left(\delta \mathcal{T}_{A}+\delta \mathcal{T}_{B}\right) \\
& +\delta \mathcal{T}_{C}\left\{\epsilon_{i \gamma n} \frac{r_{n}}{r} \cos (\overline{\mathcal{N}}+\mathcal{N})-\left(\delta_{i \gamma}-\frac{r_{i} r_{\gamma}}{r^{2}}\right) \sin (\overline{\mathcal{N}}+\mathcal{N})\right\} \\
& -\mathcal{T}_{A}\left\{\left(\delta_{i \gamma}-\frac{r_{i} r_{\gamma}}{r^{2}}\right) \sin (\overline{\mathcal{N}}+\mathcal{N})-\epsilon_{i \gamma n} \frac{r_{n}}{r} \cos (\overline{\mathcal{N}}+\mathcal{N})\right\} \delta(\overline{\mathcal{N}}+\mathcal{N}) \\
& -\mathcal{T}_{C}\left\{\epsilon_{i \gamma n} \frac{r_{n}}{r} \sin (\overline{\mathcal{N}}+\mathcal{N})+\left(\delta_{i \gamma}-\frac{r_{i} r_{\gamma}}{r^{2}}\right) \cos (\overline{\mathcal{N}}+\mathcal{N})\right\} \delta(\overline{\mathcal{N}}+\mathcal{N}) .
\end{aligned}
$$

When Eqs.(2.35), (2.36), (2.37), (2.38), and (2.46) are used to replace $\delta \mathcal{N}, \delta \overline{\mathcal{N}}, \delta \mathcal{T}_{A}, \delta \mathcal{T}_{B}$, and $\delta \mathcal{T}_{C}$ in Eq.(2.47), we find that $\delta A_{\mathrm{Gl} i}^{\gamma}(\mathbf{r})=0$, confirming the consistency of the procedure that leads to Eq.(2.46).

The effect of small gauge transformations on the limits of $\delta \overline{\mathcal{N}}$ as $u \rightarrow \pm \infty$ can best be explored by expressing Eq.(2.46) in terms of the independent variable $u$. As is discussed in the Appendix, the expression $r \mathcal{T}_{C}$ appearing on the right-hand-side of that equation is subject to the conditions $\lim _{u \rightarrow \infty}\left|r_{0} \exp (u) \mathcal{T}_{C}(u)\right|<K \exp (-\alpha u)$ and $\lim _{u \rightarrow-\infty}\left|r_{0} \exp (u) \mathcal{T}_{C}(u)\right|<$ $K^{\prime} \exp (\alpha u)$, where $K$ and $K^{\prime}$ are constants. Constraints can be placed on $\Delta$, but the only one necessary to complete this argument is that $\Delta$ is bounded in the interval $(-\infty, \infty)$. These circumstances allow us to conclude that Eq.(2.46) can be represented, in the limits $u \rightarrow \infty$ and $u \rightarrow-\infty$, in the form

$$
\left|\frac{d^{2}}{d u^{2}} \delta \overline{\mathcal{N}}(u)+\frac{d}{d u} \delta \overline{\mathcal{N}}(u)-2 \delta \overline{\mathcal{N}}(u)\right| \leq C \exp (-\alpha|u|),
$$

where $C$ is a constant. We can therefore infer that

$$
\lim _{u \rightarrow-\infty}|\delta \overline{\mathcal{N}}(u)| \leq A \exp (\alpha u)+B \exp (u) \text { and } \lim _{u \rightarrow \infty}|\delta \overline{\mathcal{N}}(u)| \leq A^{\prime} \exp (-\alpha u)+B^{\prime} \exp (-2 u)
$$

for some constants $A, B, A^{\prime}$, and $B^{\prime}$. We observe that the limiting values of $\delta \overline{\mathcal{N}}(u)$, as $u \rightarrow \pm \infty$, vanish, and that therefore the corresponding limiting values of $\overline{\mathcal{N}}(u)$, as $u \rightarrow \infty$ and as $u \rightarrow-\infty$, are invariant to small gauge transformations - i. e. transformations implemented by $\partial_{i} \Pi_{i}^{\gamma}(\mathbf{r})+g \epsilon_{\gamma \beta \alpha} A_{i}^{\beta}(\mathbf{r}) \Pi_{i}^{\alpha}(\mathbf{r})+g \psi^{\dagger}(\mathbf{r}) \frac{\tau^{\gamma}}{2} \psi(\mathbf{r})$ acting as the generator of infinitesimal gauge transformations. But $\overline{\mathcal{N}}(u)$, for arbitrary values of $u$, does not share that invariance. These arguments will be used, in the next section, to show that the winding number of the gaugeinvariant gauge field remains unaffected by small gauge transformations.

\section{TOPOLOGY AND THE IMPLEMENTATION OF GAUGE INVARIANCE}

Our considerations in this section are based on the fact, discussed in Section [1], that the transformation that converts the quark field $\psi(\mathbf{r})$ and the gauge field $A_{i}^{\gamma}(\mathbf{r})$ into their 
respective gauge-invariant forms - as shown in Eqs.(1.3) and (1.6) respectively - has the formal structure of a gauge transformation, although it differs from a gauge transformation in a very important respect: The argument of the operator-valued $V_{\mathcal{C}}(\mathbf{r})$ that effects the transformation to gauge-invariant forms is not a function by which $\psi(\mathbf{r})$ and $A_{i}^{\gamma}(\mathbf{r})$ are gaugetransformed, but is itself operator-valued and subject to gauge transformations. Eqs.(1.3) and (1.6) therefore actually represent transformations of gauge-dependent fields to forms that are invariant to further gauge transformations. Nevertheless, when we represent $V_{\mathcal{C}}(\mathbf{r})$ as a number-valued realization of the operator-valued quantity, we can use its formal structure to investigate the topological features of the gauge-invariant gauge fields.

With the ansatz that led to the expression for the resolvent field given in Eq. (2.22), we find that $V_{C}(\mathbf{r})$ is given by

$$
V_{C}(\mathbf{r})=\exp \left(-i \hat{r}_{n} \tau_{n} \frac{(\overline{\mathcal{N}}+\mathcal{N})}{2}\right) .
$$

and that the expression for $A_{\mathrm{Gl} i}^{\gamma}(\mathbf{r})$ given in Eq.(2.27) can be written as

$$
-i g \frac{\tau^{\gamma}}{2} A_{\mathrm{Gl} i}^{\gamma}(\mathbf{r})=-i g V_{C}(\mathbf{r}) \frac{\tau^{\gamma}}{2} A_{i}^{\gamma}(\mathbf{r}) V_{C}^{-1}(\mathbf{r})+V_{C}(\mathbf{r}) \partial_{i} V_{C}^{-1}(\mathbf{r})
$$

where $A_{i}^{\gamma}(\mathbf{r})$ is given in Eqs.(2.18), and where we can define

$$
\mathrm{A}_{i}(\mathbf{r})=-i g \frac{\tau^{\gamma}}{2}\left[A_{\mathrm{Gl} i}^{\gamma}(\mathbf{r})\right]_{V}=V_{C}(\mathbf{r}) \partial_{i} V_{C}^{-1}(\mathbf{r})
$$

so that $\left[A_{\mathrm{Gl} i}^{\gamma}(\mathbf{r})\right]_{V}$ is the part of $A_{\mathrm{Gl} i}^{\gamma}(\mathbf{r})$ given by

$$
\left[A_{\mathrm{Gl} i}^{\gamma}(\mathbf{r})\right]_{V}=\frac{1}{g r}\left\{\epsilon_{i \gamma n} \frac{r_{n}}{r}(\cos (\overline{\mathcal{N}}+\mathcal{N})-1)-\left(\delta_{i \gamma}-\frac{r_{i} r_{\gamma}}{r^{2}}\right) \sin (\overline{\mathcal{N}}+\mathcal{N})-\frac{r_{i} r_{\gamma}}{r} \frac{d(\overline{\mathcal{N}}+\mathcal{N})}{d r}\right\} .
$$

Eq.(3.3) shows that $\left[A_{\mathrm{GI} i}^{\gamma}(\mathbf{r})\right]_{V}$ has the formal structure of the "pure gauge" part of a gauge field, although that is not what it is. $\left[A_{\mathrm{Gl} i}^{\gamma}(\mathbf{r})\right]_{V}$ is a part of the gauge-invariant field $A_{\mathrm{Gl} i}^{\gamma}(\mathbf{r})$, and any change in $\left[A_{\mathrm{Gl} i}^{\gamma}(\mathbf{r})\right]_{V}$ due to an infinitesimal gauge transformation must be offset by compensating gauge transformations to $V_{C}(\mathbf{r}) \frac{\tau^{\gamma}}{2} A_{i}^{\gamma}(\mathbf{r}) V_{C}^{-1}(\mathbf{r})$. We can, nevertheless, make use of the formal structure of $\mathrm{A}_{i}(\mathbf{r})$ represented in Eq.(3.3) to define

$$
Q=-\left(24 \pi^{2}\right)^{-1} \epsilon_{i j k} \int d \mathbf{r} \operatorname{Tr}\left[\mathrm{A}_{i}(\mathbf{r}) \mathrm{A}_{j}(\mathbf{r}) \mathrm{A}_{k}(\mathbf{r})\right]
$$

and, following Ref. [7], we can use Eqs.(3.1) and (3.3) to express $Q$ as

$$
Q=\frac{1}{4 \pi^{2}} \int \frac{d \mathbf{r}}{r^{2}} \sin ^{2}\left(\frac{\overline{\mathcal{N}}+\mathcal{N}}{2}\right) \frac{d(\overline{\mathcal{N}}+\mathcal{N})}{d r}
$$

and integrate this expression to obtain 


$$
\begin{aligned}
Q= & \frac{1}{2 \pi} \lim _{r \rightarrow \infty}\{\overline{\mathcal{N}}(r)+\mathcal{N}(r)-\sin (\overline{\mathcal{N}}(r)+\mathcal{N}(r))\} \\
& -\frac{1}{2 \pi}\{\overline{\mathcal{N}}(0)+\mathcal{N}(0)-\sin (\overline{\mathcal{N}}(0)+\mathcal{N}(0))\}
\end{aligned}
$$

We will refer to $Q$ as the winding number of the gauge-invariant gauge field, even though its values are not restricted to integers. Unlike the winding numbers of the gauge field, which are integer-valued, [7 10] $Q$ is determined by the properties of $\overline{\mathcal{N}}$, which is governed by Eq.(2.25) and by the fact that it must be bounded in the entire interval $0 \leq r<\infty$. In discussing the properties of $\mathcal{N}$, we define the "pure gauge" gauge field

$$
A_{i}^{\gamma}(\mathbf{r})_{\mathrm{pg}}=\frac{1}{g r}\left\{\epsilon_{i \gamma n} \frac{r_{n}}{r}[\cos (\mathcal{N})-1]-\left(\delta_{i \gamma}-\frac{r_{i} r_{\gamma}}{r^{2}}\right) \sin \mathcal{N}-\frac{r_{i} r_{\gamma}}{r} \frac{d \mathcal{N}}{d r}\right\}
$$

which we obtain from

$$
A_{i}^{\gamma}(\mathbf{r})_{\mathrm{pg}}=-\frac{i}{g} \operatorname{Tr}\left[\tau_{\gamma} \exp \left(-i \mathcal{N} \frac{\tau_{\alpha} r_{\alpha}}{2 r}\right) \partial_{i} \exp \left(i \mathcal{N} \frac{\tau_{\alpha} r_{\alpha}}{2 r}\right)\right]
$$

We further observe that, in Section $\mathbb{\Pi}$ and in the Appendix, we have assumed that $\mathcal{N}=0$ at $r=0$, and that $\lim _{r \rightarrow \infty} \mathcal{N}=2 \ell \pi$, so that we consider gauge fields $A_{i}^{\gamma}(\mathbf{r})_{\mathrm{pg}}$ whose winding number is the integer $\ell$, and therefore that

$$
Q=\frac{1}{2 \pi}\left\{\lim _{r \rightarrow \infty}[\overline{\mathcal{N}}(r)-\sin (\overline{\mathcal{N}}(r))]+2 \pi \ell-\overline{\mathcal{N}}(0)+\sin (\overline{\mathcal{N}}(0))\right\} .
$$

As is shown in the Appendix, when $\ell=0$ and $\overline{\mathcal{N}}$ is normalized so that $\overline{\mathcal{N}}=0$ when $r=0$, $\lim _{r \rightarrow \infty} \overline{\mathcal{N}}=m \pi$. For the $\ell=0$ case, $\mathrm{Q}$ is therefore given by

$$
Q=\frac{1}{2 \pi}\left(\lim _{r \rightarrow \infty} \overline{\mathcal{N}}(r)-\overline{\mathcal{N}}(0)\right)=\frac{1}{2 \pi} \lim _{r \rightarrow \infty} \overline{\mathcal{N}}(r)=\frac{m}{2}
$$

where $m$ is an integer. We will now discuss the implications of Eq.(2.25) for the values of $Q$ when $\ell \neq 0$.

When we consider gauge fields with winding numbers $\ell \neq 0$, for which $\lim _{u \rightarrow \infty} \mathcal{N}(u)=2 \pi \ell$, but $\lim _{u \rightarrow \infty} \exp (u) \mathcal{T}_{A}(u)$, and $\lim _{u \rightarrow \infty} \exp (u) \mathcal{T}_{C}(u)$ still vanish along with $\lim _{u \rightarrow-\infty} \mathcal{N}(u)$, $\lim _{u \rightarrow-\infty} \exp (u) \mathcal{T}_{A}(u)$, and $\lim _{u \rightarrow-\infty} \exp (u) \mathcal{T}_{C}(u)$, then we observe that, in the limit $u \rightarrow \infty$, $\overline{\mathcal{N}}(u)$ satisfies

$$
\frac{d^{2} \overline{\mathcal{N}}}{d u^{2}}+\frac{d \overline{\mathcal{N}}}{d u}+2[2 \pi \ell \cos (\overline{\mathcal{N}}+2 \pi \ell)-\sin (\overline{\mathcal{N}}+2 \pi \ell)]=0
$$

and $\overline{\mathcal{N}}(u)$ approaches a limiting value $\lim _{u \rightarrow \infty} \overline{\mathcal{N}}(u)$, which exists, but is not an integer multiple of $\pi . \overline{\mathcal{N}}(u)$ vanishes, as before, at the saddle point for which $u \rightarrow-\infty$, but $\lim _{u \rightarrow \infty} \overline{\mathcal{N}}(u)$ now is one of the denumerably infinite solutions of the transcendental equation

$$
\tan \left\{\lim _{u \rightarrow \infty} \overline{\mathcal{N}}(u)\right\}=2 \pi \ell .
$$

If we adopt the convention that the inverse tangent is defined so that, for $\xi=\tan ^{-1}(\eta)$, $-\frac{\pi}{2}<\xi<\frac{\pi}{2}$, then the winding numbers for the gauge-invariant gauge field are given by 


$$
Q=\frac{1}{2 \pi}\left\{\tan ^{-1}(2 \pi \ell)+m \pi+2 \pi \ell\left(1-\frac{1}{\sqrt{1+4 \pi^{2} \ell^{2}}}\right)\right\}
$$

for some integer $m$.

Eq.(3.14) establishes a winding number for the gauge-invariant gauge field that is a function of two integer-valued variables, $\ell$ and $m$, but that is not itself integer-valued. The integer $\ell$ defines the homotopy class of the gauge field described by Eqs.(3.8) and (3.9). Gauge-invariant gauge fields can be categorized by the value of $\ell$ that describes the homotopy class of the gauge fields to which they are linked by Eq.(2.25), and by the value of $m$ that defines the sheet on which the inverse tangent function that corresponds to the $\lim _{u \rightarrow \infty} \overline{\mathcal{N}}(u)$ is located. The range of values of the integer $m$, for which $m \pi$ corresponds to limits that $\overline{\mathcal{N}}(u)$ can actually attain, depends on the solutions of Eq.(2.25) that given sets of functions $\mathcal{N}(u), \mathcal{T}_{A}(u)$ and $\mathcal{T}_{C}(u)$ can support. Examples of solutions, and their implications for winding numbers of the gauge-invariant gauge field, are given in Section $\mathbb{\nabla}$.

The relationship between $Q, \ell$ and $m$ reflects the fact that, when $\lim _{u \rightarrow-\infty} \mathcal{N}(u)=0$, the $\lim _{u \rightarrow \infty} \mathcal{N}(u)$ is the only part of the gauge field that can affect its homotopy class; however, the functional dependence of $\mathcal{N}(u), \mathcal{T}_{A}(u)$ and $\mathcal{T}_{C}(u)$ for the entire range of values of $u$ affects the $\lim _{u \rightarrow \infty} \overline{\mathcal{N}}(u)$, and therefore $Q$, as shown in Eqs.(2.25) and (3.10). A number of different values of $m$ can therefore be compatible with the same $\ell$, and, in fact, with the identical functions $\mathcal{N}(u), \mathcal{T}_{A}(u)$ and $\mathcal{T}_{C}(u)$. The mathematical foundations for these observations are discussed in Section $\mathbb{D}$ and in the Appendix.

\section{ILLUSTRATIVE NUMERICAL EXAMPLES}

In this section, we present illustrative examples of numerical integrations of Eq.(2.25) that simulate solutions that are bounded for all real values of the independent variable. In carrying out these numerical integrations, we have selected functional forms for $\mathcal{T}_{A}, \mathcal{T}_{C}$, and $\mathcal{N}$ that are consistent with assumptions (A1) to (A3) and that include examples of $\mathcal{N}$ that belong to the trivial homotopy class with $\ell=0$, as well as others in which $\lim _{t \rightarrow \infty} \mathcal{N}=2 \pi \ell$ with $\ell \neq 0$. The technical considerations on which these simulations are based, and the individual examples, constitute the remainder of this section

In Theorem 1 in the Appendix, we have established the existence of a solution $\overline{\mathcal{N}}$ satisfying equation (A4) and boundary conditions (A5), provided assumptions (A1) to (A3) are satisfied. (It is noted that $w$ and $y$ in the Appendix represent $\mathcal{N}$ and $\overline{\mathcal{N}}$, respectively.) Such assumptions essentially say that $e^{t} \mathcal{T}_{A}$ and $e^{t} \mathcal{T}_{C}$ behave like 0 for large $|t|$, and that $\mathcal{N}$ behaves like 0 as $t \rightarrow-\infty$, and like $2 \ell \pi$ for some integer $\ell$, as $t \rightarrow \infty$.

\footnotetext{
${ }^{2}$ In this section, and in the Appendix, Eq.(2.25) will sometimes be written in the form of Eq.(A4), and the independent variable $u$ will sometimes be written as $t$.
} 
Remark 3 in Theorem 1 asserts that there are an infinite number of solutions to Eq.(A4). But the question arises: Will numerical solutions of Eq.(A4), that are bounded in the entire interval $(-\infty, \infty)$ and normalized so that $\lim _{t \rightarrow-\infty} \overline{\mathcal{N}}=0$, always settle down to the same two values of $m$ as in the case for the damped pendulum? (It is well known, in the case of Eq.(2.26) which describes a damped pendulum, that there are exactly two classes of solutions - one for $m=1$ and another for $m=-1$.) We will carry out some numerical experiments to address this question, and document some of our results in this section.

Since our numerical computations will be done on a finite interval $[-M, M]$ for some large value $M>0$, we will first construct the appropriate boundary condition at $t=-M$. With the solution $\overline{\mathcal{N}}$ going to zero as $t \rightarrow-\infty$, one can impose the boundary condition $\overline{\mathcal{N}}(-M)=0$. However, a better boundary condition can be constructed, which leads to reduced numerical errors even when we use only a moderate value of $M$. This reduced interval size for $[-M, M]$ leads to more efficient and more accurate calculations. Such attributes of numerical infinity are well known (see Chapter 4, Ref. [14).

Since both $\overline{\mathcal{N}}$ and $\mathcal{N}$ tend to zero as $t$ approaches $-\infty$, we employ a Taylor's expansion to extract the leading order behavior of the terms in equation (A4) in that regime. We keep only the leading order terms in $\mathcal{N}$ and $\overline{\mathcal{N}}$, taking into account that while both are small when $t \rightarrow-\infty$, the relative magnitudes of $\overline{\mathcal{N}}$ and $\mathcal{N}$ are not known. In this way, we find that

$$
\mathcal{N} \cos (\overline{\mathcal{N}}+\mathcal{N})-\sin (\overline{\mathcal{N}}+\mathcal{N}) \approx-\overline{\mathcal{N}}-\mathcal{N}^{3} / 3 .
$$

Hence from (A4), we expect that Eq.(2.25) is well approximated by

$$
\overline{\mathcal{N}}^{\prime \prime}+\overline{\mathcal{N}}^{\prime}-2 \overline{\mathcal{N}}-2 \mathcal{N}^{3} / 3-\beta e^{t} \mathcal{T}_{A} \mathcal{N}^{2} / 2-\beta e^{t} \mathcal{T}_{C} \mathcal{N} \approx 0 .
$$

where ' designates differentiation with respect to $t$. The relative magnitudes of the three source terms containing $\mathcal{N}$ in the above equation as $t \rightarrow-\infty$, will determine which one will dominate over the others. Assuming that the dominant behavior of these three source terms is $\gamma e^{\omega t}$ for some constants $\gamma$ and $\omega>0$ as $t$ approaches $-\infty$, we can further simplify Eq.(1.2) and obtain the following linear non-homogeneous equation with constant coefficients:

$$
\overline{\mathcal{N}}^{\prime \prime}+\overline{\mathcal{N}}^{\prime}-2 \overline{\mathcal{N}}+\gamma e^{\omega t} \approx 0 .
$$

If we had exact equality in the above equation, the complementary solutions would be spanned by $e^{-2 t}$ and $e^{t}$. A particular solution of such an equation would be of the form $\gamma_{1} e^{\omega t}$, for some constant $\gamma_{1}$. Since $\overline{\mathcal{N}}$ is bounded as $t \rightarrow-\infty$, we expect solutions for large negative $t$ to be of the form

$$
\overline{\mathcal{N}} \approx C e^{t}+\gamma_{1} e^{\omega t}
$$

for some constant $C$. The following two sets of circumstances may apply:

Case I: $\omega>1$.

In this case, $\overline{\mathcal{N}} \approx C e^{t}$. In order to avoid the unbounded complementary solution $e^{-2 t}$, we will employ the boundary condition $\overline{\mathcal{N}}^{\prime}(-M)=\overline{\mathcal{N}}(-M)$. We can assign an arbitrary 
value, $\delta$, to both $\overline{\mathcal{N}}$ and to $\overline{\mathcal{N}}^{\prime}$ at $t=-M$, so long as $M$ is sufficiently large and $|\delta|$ is sufficiently small. Thus we can employ a standard initial value problem-solver (like Runge Kunta method with local error control, as can be found in any standard software) to solve equation (A4), and integrate Eq.(2.25) from $t=-M$ to a large positive value of $t$. We can also integrate backwards from $t=-M$ to a negative value of $t$ whose absolute value is even larger than $M$.

Case II: $\omega<1$.

$\overline{\mathcal{N}} \approx C e^{\omega t}$. In order to avoid the unbounded complementary solution $e^{-2 t}$ in this case, we would have to employ the boundary condition $\overline{\mathcal{N}}^{\prime}(-M)=\omega \overline{\mathcal{N}}(-M)$.

In all the numerical experiments presented in the following illustrative examples, the dominant behavior as $t \rightarrow-\infty$ is that of the complementary solution $e^{t}$, so that all our examples belong to case I. We therefore impose the initial conditions $\overline{\mathcal{N}}^{\prime}(-M)=\overline{\mathcal{N}}(-M)=\delta$, for some large $M>0$ and small $|\delta|$ in these experiments. Provided such conditions are met, when we integrate equation (A4) backward in $t$ towards $-\infty$, numerical solutions of $\overline{\mathcal{N}}$ stay close to the value 0 for a long interval. This will be clearly seen in Fig. 1 to Fig. 6 below. Since we also have an unbounded mode $e^{-2 t}$ near $t=-\infty, \overline{\mathcal{N}}=0$ acts like a saddle point. Hence if we continue to integrate backward in time, the numerical solution will eventually blow up, as one can never get rid of the unbounded mode entirely in numerical calculations, even though Theorem 1 demonstrates that solutions exist that never deviate from 0 as $t \rightarrow-\infty$.

Should we use conditions other than $\overline{\mathcal{N}}^{\prime}(-M)=\overline{\mathcal{N}}(-M)$, the magnitude of the solutions will become large very quickly as we integrate backward in the variable $t$. A larger value of $M$ is needed to ensure that condition (A5) is satisfied approximately in such a scenario. This larger domain will increase the computational cost and decrease the accuracy of the simulation results.

From the proof of Theorem 1, we know that for any value of $\delta$ and $M$, a solution $\overline{\mathcal{N}}$ will settle down to a value as depicted by condition (A5b). Hence we expect that there are infinitely many solutions to Eq.(A4) satisfying boundary conditions (A5). But the existence of an infinity of solutions does not imply that there are infinitely many $m$ values that these solutions approach as $t \rightarrow \infty$. We have carried out numerous numerical experiments to address this question. These experiments show that solutions of Eq.(A4) exist, for the identical $\mathcal{T}_{A}, \mathcal{T}_{C}$, and $\mathcal{N}$, for which $\lim _{t \rightarrow-\infty} \overline{\mathcal{N}}=0$, which demonstrate that $\overline{\mathcal{N}}$ approaches limits corresponding to at least three different values of $m$ as $t \rightarrow \infty$. We will document some of the more interesting numerical results below:

\section{A. Experiment 1}

In this experiment we have used

$$
\mathcal{N}=\frac{10 e^{t}}{\left(1+e^{2 t}\right)^{2}} \quad \text { and } \quad \mathcal{T}_{A}=\mathcal{T}_{C}=t^{3} \exp \left(-3 t^{2}\right)\left(1-\exp \left(-6 t^{2}\right)\right)
$$


with $\beta=2 g r_{0}=250$, where we have taken $-M$ as the value of negative numerical infinity with $M=13.03423$. We have set $\overline{\mathcal{N}}=\overline{\mathcal{N}}^{\prime}=10^{-6}$ at $t=-M$. The result, using a standard initial value problem-solver, is represented in Figure 1. We observe that $\overline{\mathcal{N}}$ is essentially zero from about $t=-5$ to $t=-25\left(|\overline{\mathcal{N}}(-25)| \approx 10^{-5}\right)$, that it hovers near the unstable equilibrium position $\overline{\mathcal{N}}=6 \pi$ to within better than 1 part per $10^{5}$ at $t=10$, and stabilizes to its final position at $\overline{\mathcal{N}}=7 \pi$. With reference to Eq.(3.14), $\ell=0$ and $m=7$. The winding number for the gauge-invariant gauge field in this numerical experiment is $Q=\frac{7}{2}$.

\section{B. Experiment 2}

We employ exactly the same data here as in Experiment 1, except that in our choice of numerical infinity $-M$ in this experiment, $M=13.034$; and that we have set $\overline{\mathcal{N}}=\overline{\mathcal{N}}^{\prime}=$ $-10^{-6}$ at $t=-M$. The result is represented in Figure 2 . We observe in this case that $\overline{\mathcal{N}}$ is again essentially zero from about $t=-5$ to $t=-25\left(|\overline{\mathcal{N}}(-25)| \approx 10^{-5}\right)$, that it briefly hovers near the unstable equilibrium position $\overline{\mathcal{N}}=6 \pi$ in the vicinity of $t=8$, and stabilizes to its final position at $\overline{\mathcal{N}}=5 \pi$. With reference to Eq.(3.14), $\ell=0$ and $m=5$. The winding number for the gauge-invariant gauge field in this numerical experiment is $Q=\frac{5}{2}$.

\section{Experiment 3}

We again employ exactly the same data here as in Experiments 1 and 2, except that in our choice of numerical infinity $-M$ in this case $M=30$, and that we have set $\overline{\mathcal{N}}=\overline{\mathcal{N}}^{\prime}=10^{-6}$ at $t=-M$. The result is represented in Figure 3. We observe in this case that $\overline{\mathcal{N}}$ is again essentially zero from about $t=-5$ to $t=-25\left(|\overline{\mathcal{N}}(-40)|<2 \times 10^{-10}\right)$, that it remains within approximately $1 \%$ of $\pi$ in the vicinity of $t=-9$, and that it stabilizes to its final position at $\overline{\mathcal{N}}=9 \pi$. With reference to Eq.(3.14), $\ell=0$ and $m=9$. The winding number for the gauge-invariant gauge field in this numerical experiment is $Q=\frac{9}{2}$.

\section{Experiment 4}

In this experiment we have used

$$
\mathcal{N}=\frac{100 e^{t}}{\left(1+e^{2 t}\right)^{2}}+\left(1-\frac{1}{1+e^{t}}\right) 2 \pi \quad \text { and } \quad \mathcal{T}_{A}=\mathcal{T}_{C}=t^{3} \exp \left(-3 t^{2}\right)\left(1-\exp \left(-6 t^{2}\right)\right)
$$

with $\beta=2 g r_{0}=250$, and we have taken $-M$ as the value of negative numerical infinity, with $M=20$. We have set $\overline{\mathcal{N}}=\overline{\mathcal{N}}^{\prime}=-10^{-4}$ at $t=-M$. The result is represented in Figure 4. We observe that $\overline{\mathcal{N}}$ is essentially zero from about $t=-12$ to $t=-30\left(|\overline{\mathcal{N}}(-30)| \approx 10^{-5}\right)$; that it briefly hovers near $\overline{\mathcal{N}}=-\pi$ from approximately $t=-8$ to $t=-6$ to within about $.2 \%$, and that it stabilizes to its final position at $\overline{\mathcal{N}}=29.6874$. Since $\tan ^{-1}(2 \pi)=1.41297$, and $29.6874-1.41297=28.2744=9 \pi$, this experiment exemplifies an $\ell=1$ and $m=9$ case. The winding number for the gauge-invariant gauge field in this numerical experiment is $Q=5.5677$. 


\section{E. Experiment 5}

We employ exactly the same data here as in Experiment 4, but choose $M=15$. In this experiment, we have set $\overline{\mathcal{N}}=\overline{\mathcal{N}}^{\prime}=-10^{-4}$ at $t=-M$. The result is represented in Figure 5. We observe in this case that $|\overline{\mathcal{N}}(-25)| \approx 10^{-5}$, and that it stabilizes to its final position at $\overline{\mathcal{N}}=17.1214$. Since $\tan ^{-1}(2 \pi)=1.41297$, and $17.1214-1.41297=15.7084=5 \pi$, this experiment exemplifies an $\ell=1$ and $m=5$ case. The winding number for the gaugeinvariant gauge field in this numerical experiment is $Q=3.5677$.

\section{F. Experiment 6}

We employ exactly the same data here as in Experiments 4 and 5 , but choose $M=30$. In this experiment, we have set $\overline{\mathcal{N}}=\overline{\mathcal{N}}^{\prime}=10^{-4}$ at $t=-M$. The result is represented in Figure 6. We observe in this case that $|\overline{\mathcal{N}}(-35)| \approx 10^{-6}$, that it hovers near $\overline{\mathcal{N}}=\pi$ from approximately $t=-17$ to $t=-6$ to about $3 \%$ and that it stabilizes to its final position at $\overline{\mathcal{N}}=35.9699$. Since $\tan ^{-1}(2 \pi)=1.41297$, and $35.9699-1.41297=34.557=11 \pi$, this experiment exemplifies an $\ell=1$ and $m=11$ case. The winding number for the gaugeinvariant gauge field in this numerical experiment is $Q=6.5677$.

\section{G. Comments on Numerical Experiments}

We note that in all six experiments, we were able to calculate backward in $t$ from $t=-M$ for a long range, and still could obtain small values of $\overline{\mathcal{N}}$. This shows that our choice of initial conditions $\overline{\mathcal{N}}^{\prime}(-M)=\overline{\mathcal{N}}(-M)$ has been effective and reliable.

With reference to Theorem 11, we note that in Experiments 1, 2 and 3, the given $\mathcal{N}$ leads to $\ell=0$ for the limit specified in Assumption A2; similarly, in Experiments 4, 5 and 6, the given $\mathcal{N}$ leads to $\ell=1$. Hence, for large $t$, in Experiments 1,2 and $3, \overline{\mathcal{N}}$ converges to $m \pi$ for some integer $m$, and, in Experiments 4,5 and $6, \overline{\mathcal{N}}$ converges to $\tan ^{-1}(2 \pi)+m \pi$ for some integer $m$, in accordance with Remark 2 in the Appendix. We observe that for each of two values of $\ell, m$ is a different integer in each experiment. Thus the numerical results show that there are cases in which, for the same $\mathcal{N}, \mathcal{T}_{A}$, and $\mathcal{T}_{C}, \overline{\mathcal{N}}$ can converge to at least three different values of $m$.

A linearization analysis shows that if $m$ is odd, then $m \pi$ behaves like a stable equilibrium point. However, when $m$ is even, $m \pi$ acts like a saddle point. (That is why we have a saddle point behavior at $t=-\infty$, since it corresponds to $m=0$, which is even.) A good analogy can again be made to the damped pendulum case. The even values of $m$ correspond to an inverted pendulum whose equilibrium is unstable, while the odd values correspond to the lowest stable equilibrium point. While it is theoretically possible for a moving pendulum to stop exactly at the inverted position, any slight error will prevent us from observing such a phenomenon in numerical calculations. (This is equivalent to observing that we can never completely eliminate the unbounded mode in numerical calculations.) However, in principle, 
there is no reason why $\overline{\mathcal{N}}$ cannot approach an unstable limit point at which $m$ is even, as $t \rightarrow \infty$. In fact, Experiments 1 and 2 substantiate this claim, since the solutions $\overline{\mathcal{N}}$ hover near $6 \pi$ for such an extended interval. It is not surprising to find that the precise values of initial conditions that bring about the transition from $5 \pi$ to $7 \pi$ are sensitive to the numerical accuracy of the computation. In Experiments 1 and 2, a change in the numerical accuracy of the computation can require a $1 \%$ change in the value of $M$ to obtain similar qualitative results. 


\section{FIGURES}

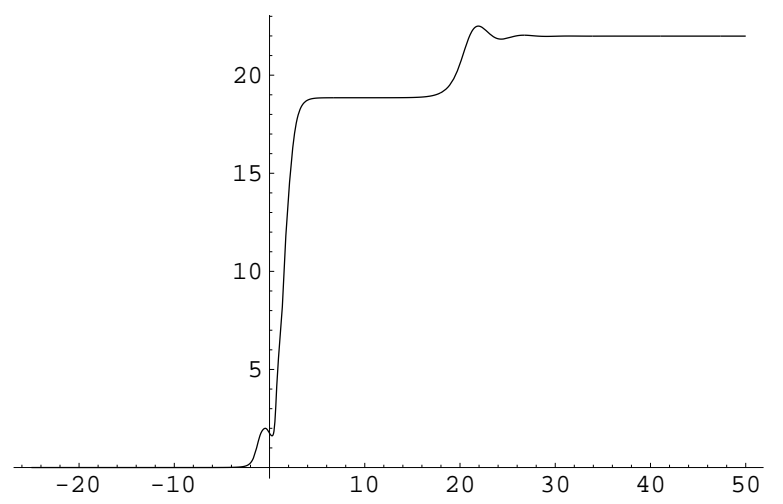

FIG. 1.

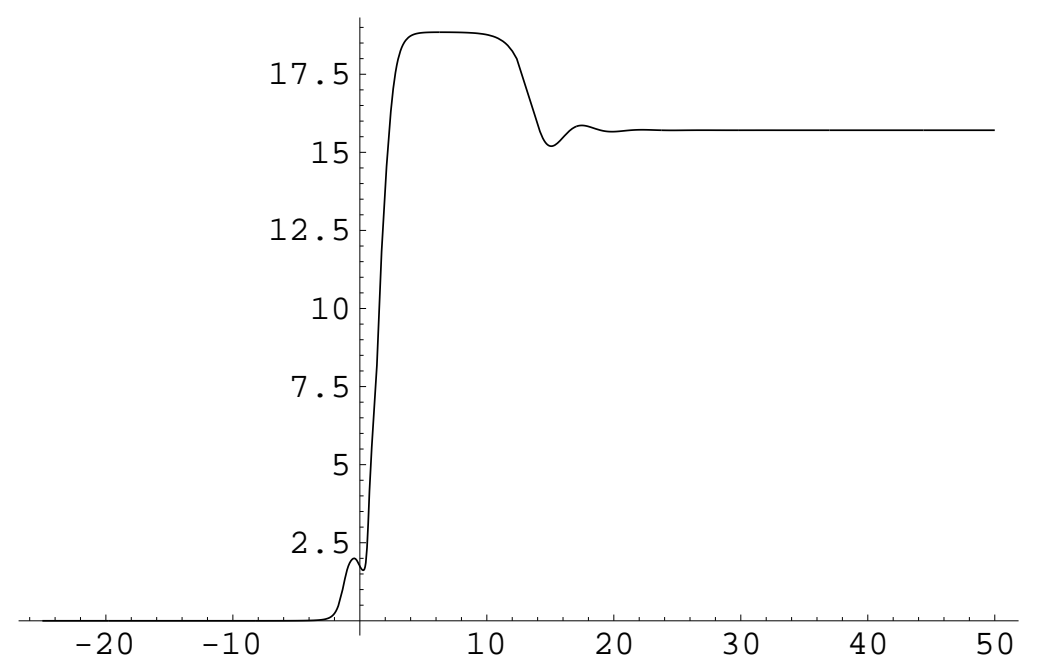

FIG. 2.

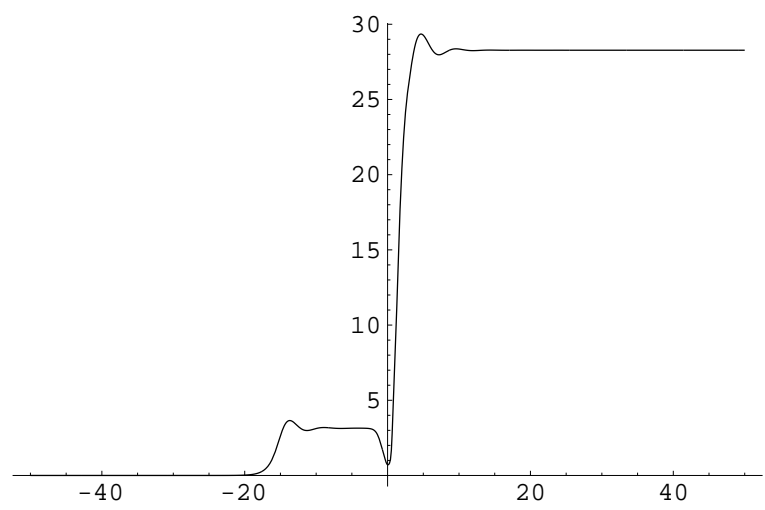

FIG. 3. 


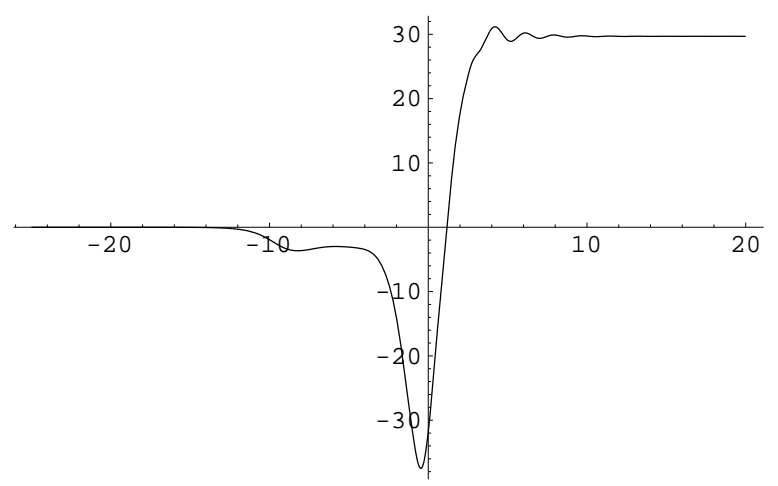

FIG. 4.

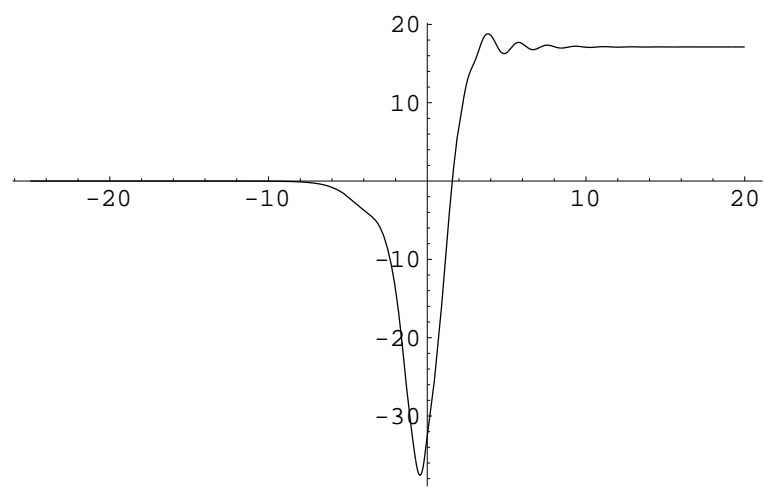

FIG. 5 .

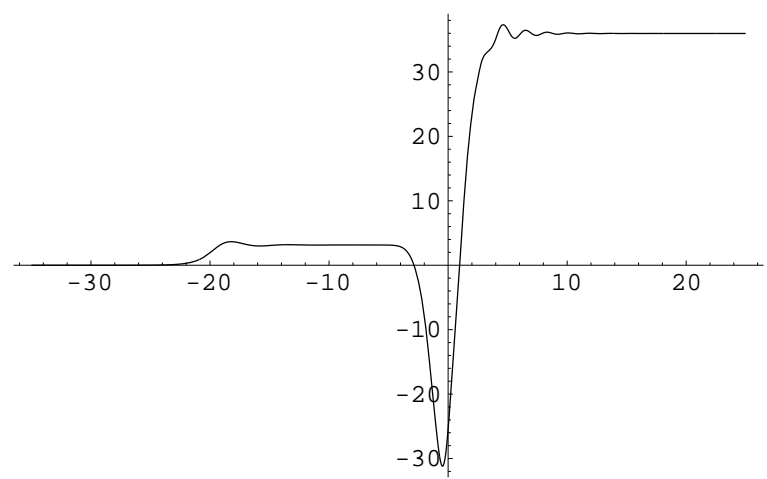

FIG. 6 .

\section{ACKNOWLEDGMENTS}

One of us (KH) thanks Prof. Gerald Dunne for a number of helpful conversations. The work of K. Haller and L. Chen was supported by the Department of Energy under Grant No. DE-FG02-92ER40716.00. The work of Y. S. Choi was partially supported by NIH grant 1P41-RR13186-01. 


\section{APPENDIX A:}

In Section III, the discussion is based on the properties of the solutions of Eq.(2.25). The theorem that establishes these solutions is proven in this Appendix. In this proof, the function $\overline{\mathcal{N}}$ and $\mathcal{N}$ are represented as $y$ and $w$ respectively, and the independent variable $u$ is represented as $t$.

Let $\mathcal{T}_{A}, \mathcal{T}_{C}$, and $w$ be given $C^{1}$ (continuously differentiable) functions of $t$ in the interval $(-\infty, \infty)$, let $\beta$ be a given positive constant, and let $\ell$ be a given integer. We assume that there is a positive constant $\alpha$ such that

$$
\begin{aligned}
& \lim _{t \rightarrow-\infty} e^{(1-\alpha) t} \mathcal{T}_{A}=0, \quad \lim _{t \rightarrow-\infty} e^{(1-\alpha) t} \mathcal{T}_{C}=0, \quad \lim _{t \rightarrow-\infty} e^{-\alpha t} w=0, \\
& \lim _{t \rightarrow \infty} e^{(1+\alpha) t} \mathcal{T}_{A}=0, \quad \lim _{t \rightarrow \infty} e^{(1+\alpha) t} \mathcal{T}_{C}=0, \quad \lim _{t \rightarrow \infty} e^{\alpha t}(w-2 \ell \pi)=0,
\end{aligned}
$$

and

$$
\lim _{t \rightarrow \infty} e^{t} \mathcal{T}_{A}^{\prime}, \lim _{t \rightarrow \infty} e^{t} \mathcal{T}_{C}^{\prime}, \quad \text { and } \lim _{t \rightarrow \infty} w^{\prime \prime} \text { exist, } \quad \lim _{t \rightarrow \infty} e^{\alpha t} w^{\prime}=0
$$

(i.e. the functions $w, e^{t} \mathcal{T}_{A}$ and $e^{t} \mathcal{T}_{C}$ are exponentially decaying at both positive and negative infinity.) Consider the equation

$$
\begin{aligned}
& \frac{d^{2} y}{d t^{2}}+\frac{d y}{d t}+2\{w \cos (y+w)-\sin (y+w)\} \\
& +\beta e^{t}\left\{\mathcal{T}_{A}(\cos (y+w)-1)-\mathcal{T}_{C} \sin (y+w)\right\}=0 .
\end{aligned}
$$

We have the following theorem.

Theorem 1 Under the assumptions (A1) to (A3) on $w, \mathcal{T}_{A}$ and $\mathcal{T}_{C}$ stated above, there is a solution $y$ to equation (A4) on the interval $(-\infty, \infty)$. Moreover,

$$
\text { (a) } \lim _{t \rightarrow-\infty} y(t)=2 n \pi \text {, and (b) } \lim _{t \rightarrow \infty} y(t)=\tan ^{-1}(2 \ell \pi)+m \pi \text {, }
$$

where $n$ and $m$ are integers and $\tan ^{-1}: \mathbf{R} \rightarrow\left(-\frac{\pi}{2}, \frac{\pi}{2}\right)$.

Remark 1: By using the substitution $Y=y-2 n \pi$, it suffices to prove the above theorem when $n=0$.

Remark 2: When $\ell=0$, then equation (A5b) yields $\lim _{t \rightarrow \infty} y(t)=m \pi$ for some integer $m$. When $\ell \neq 0$, then equation (A5b) yields $\lim _{t \rightarrow \infty} y(t)=\tan ^{-1}(2 \ell \pi)+m \pi$ for some integer $m$.

Remark 3: Actually the proof shows that there are an infinite number of solutions to our problem.

Proof: Because of the Remark 1, we let $n=0$. As $t \rightarrow-\infty$, we expect that the contributions from the $w, \mathcal{T}_{A}$ and $\mathcal{T}_{C}$ terms are negligible due to the assumptions (A1), so that $y=0$ behaves exactly like the unstable equilibrium point in the damped pendulum case. We now prove that this intuition is indeed what happens. 
We rewrite the equation as

$$
y^{\prime \prime}+y^{\prime}-2 y+f(t, y)=0
$$

where

$$
f(t, y) \equiv 2\{(y-\sin (y+w))+w \cos (y+w)\}+\beta e^{t}\left\{\mathcal{T}_{A}(\cos (y+w)-1)-\mathcal{T}_{C} \sin (y+w)\right\}
$$

It is noted that $|f(t, 0)| \leq o(1) e^{\alpha t}$ and $f_{y}(t, y)=o(1)$, when $t$ is large and negative and $|y|$ is sufficiently small. Next we convert it to a system of two equations,

$$
\begin{aligned}
& y^{\prime}=z, \\
& z^{\prime}=-z+2 y-f(t, y) .
\end{aligned}
$$

If, besides the conditions that $f$ satisfies, $f(t, 0)$ also were 0 for all $t$, a standard theorem in ordinary differential equations (theorem 4.1, p.330, Ref. [15] ) would enable us to conclude that, for any negative $t_{0}$ with sufficiently large modulus, there is a one dimensional manifold $S$, which depends on $t_{0}$, in the phase space $\left(y, y^{\prime}\right)$, such that if the initial conditions $y\left(t_{0}\right)$ and $y^{\prime}\left(t_{0}\right)$ lie on $S$, then $y \rightarrow 0$ as $t \rightarrow-\infty$. In our case, we have $|f(t, 0)| \leq o(1) e^{\alpha t}$ for large and negative $t$. The same proof given in theorem 4.1 in Ref. [15] still works. The only alteration needed is in the first step, when we iterate Eq. (4.11) in that theorem, since $f(t, 0)$ is not zero anymore. However, because of the exponential decay of $f(t, 0)$ for large negative $t$, Eq. (4.12) in that proof in Ref. [15] will still be valid, and the same proof stands. Fix $t_{0}$. We have thus constructed a solution $y$ to equation (A4) on the interval $\left(-\infty, t_{0}\right]$ satisfying (A5a). (Because of the one dimensional manifold $S$, this gives rise to Remark 3.)

Take any $M>0$. On any fixed bounded interval $\left[t_{0}, M\right]$,

$$
\left|2\{w \cos (y+w)-\sin (y+w)\}+\beta e^{t}\left\{\mathcal{T}_{A}(\cos (y+w)-1)-\mathcal{T}_{C} \sin (y+w)\right\}\right|
$$

is uniformly bounded for all $y$. Thus the solution $y$ can never blow up in finite time, and exists for all $t \in(-\infty, \infty)$.

To finish the proof of this theorem, it suffices to show that the solution has property (A5b). For $\ell=0$, we expect that this is the case physically, because for large $t$ our equation behaves like a damped pendulum moving under the influence of gravity only — because of assumptions (A2) and (A3) - hence we expect that the solution will settle down in an equilibrium point, i.e. $y=m \pi$ for some integer $m$. To prove the property (A5]b) for arbitrary integer values of $\ell$, take $t_{1}>0$ sufficiently large so that for $t \geq t_{1}, w \leq e^{-\alpha t}, e^{t}\left|\mathcal{T}_{A}\right| \leq e^{-\alpha t} / 2$, and $e^{t}\left|\mathcal{T}_{C}\right| \leq e^{-\alpha t} / 2$. Now for $t \geq t_{1}$, using the Cauchy-Schwarz's inequality, 


$$
\begin{aligned}
& \frac{d}{d t}\left[\frac{\left(y^{\prime}\right)^{2}}{2}+2 \cos (y+w)+2 w \sin (y+w)\right] \\
= & y^{\prime}\left(y^{\prime \prime}+2 w \cos (y+w)-2 \sin (y+w)\right)+2 w w^{\prime} \cos (y+w) \\
= & -\left(y^{\prime}\right)^{2}-\beta \exp (t)\left\{\mathcal{T}_{A}(\cos (y+w)-1)-\mathcal{T}_{C} \sin (y+w)\right\} y^{\prime} \\
& \quad+2 w w^{\prime} \cos (y+w) \\
\leq & -\left(y^{\prime}\right)^{2}+2 \beta e^{t}\left|y^{\prime}\right|\left(\left|\mathcal{T}_{A}\right|+\left|\mathcal{T}_{C}\right|\right)+2|w|\left|w^{\prime}\right| \\
\leq & -\frac{\left(y^{\prime}\right)^{2}}{2}+4 \beta^{2}\left[e^{t}\left(\left|\mathcal{T}_{A}\right|+\left|\mathcal{T}_{C}\right|\right)\right]^{2}+2 C e^{-\alpha t} \\
\leq & -\frac{\left(y^{\prime}\right)^{2}}{2}+8\left(\beta^{2}+C\right) e^{-\alpha t}
\end{aligned}
$$

for some constant $C>0$. In other words, if we define $H \equiv \frac{\left(y^{\prime}\right)^{2}}{2}+2 \cos (y+w)+2 w \sin (y+$ $w)+\frac{8\left(\beta^{2}+C\right) e^{-\alpha t}}{\alpha}$, then

$$
\frac{d H}{d t} \leq-\frac{\left(y^{\prime}\right)^{2}}{2} \leq 0
$$

Hence $H$ is decreasing in $t$ for $t \geq t_{1}$, and $H \geq-2-2\|w\|_{\infty}$. Since $\frac{\left(y^{\prime}(t)\right)^{2}}{2} \leq H\left(t_{1}\right)+2+2\|w\|_{\infty}$ for $t \geq t_{1},\left|y^{\prime}\right|$ is bounded for $t \in\left[t_{1}, \infty\right)$. From the governing equation (A4), $\left|y^{\prime \prime}\right|$ is then bounded for $t \in\left[t_{1}, \infty\right)$.

Next take the derivate of the equation (A4). From this equation, we have $\left|y^{\prime \prime \prime}\right|$ is bounded for $t \in\left[t_{1}, \infty\right)$ due to assumptions (A3). We can now conclude that both $\left|H^{\prime}\right|$ and $\left|H^{\prime \prime}\right|$ are bounded for $t \in\left[t_{1}, \infty\right)$ by some simple calculations.

Since $H$ is monotone decreasing and has a lower bound, there exists a constant $H_{0}$ such that $\lim _{t \rightarrow \infty} H=H_{0}$. Together with the bound on $\left|H^{\prime \prime}\right|$, we can conclude (p.116, Ref. [16]) that $\lim _{t \rightarrow \infty} H^{\prime}=0$. It can be checked that

$$
\begin{aligned}
\frac{d H}{d t}=- & \left(y^{\prime}\right)^{2}-\beta \exp (t)\left\{\mathcal{T}_{A}(\cos (y+w)-1)-\mathcal{T}_{C} \sin (y+w)\right\} y^{\prime} \\
& +2 w w^{\prime} \cos (y+w)-8\left(\beta^{2}+C\right) e^{-\alpha t} .
\end{aligned}
$$

Taking the limit as $t \rightarrow \infty$, we have

$$
\lim _{t \rightarrow \infty}\left(y^{\prime}\right)^{2}=0
$$

which means $y^{\prime} \rightarrow 0$ as $t \rightarrow \infty$. Together with $\left|y^{\prime \prime \prime}\right|$ being bounded, this yields $\lim _{t \rightarrow \infty} y^{\prime \prime}=0$ (by the same theorem in Ref. [16]). Finally, taking the limit $t \rightarrow \infty$ in equation (A4), we obtain the result

$$
\lim _{t \rightarrow \infty}(w \cos (y+w)-\sin (y+w))=0 .
$$

This can be reduced to $\lim _{t \rightarrow \infty}[2 \ell \pi \cos y-\sin y]=0$. This is equivalent to (A5]b). The proof of the theorem is now complete. 


\section{REFERENCES}

[1] L. Chen, M. Belloni and K. Haller, Phys. Rev. D 55, 2347 (1997).

[2] K. Haller, and L. Chen, "Nonlocal Color Interactions in a Gauge-Invariant Formulation of QCD" in Proceedings of the XXIX International Conference on High Energy Physics A. Astbury, D. Axen and J. Robinson, Editors; World Scientific Publishing Co. (Singapore 1999); p. 1650

[3] K. Haller, Gauge-Invariant QCD - an Approach to Long-Range Color-Confining Forces, hep-th/9808028; to be published in the Proceedings of the Fourth International Workshop on QCD and Hadronic Physics.

[4] M. Belloni, L. Chen and K. Haller, Phys. Lett. B 403, 316 (1997).

[5] L. Chen and K. Haller Quark Confinement and Color Transparency in a Gauge-Invariant Formulation of QCD, hep-th/9803250; to be published in Int. J. Mod. Phys. A.

[6] N. H. Christ and T. D. Lee, Phys. Rev. D22 (1980), 939

[7] R. Jackiw, "Topological Investigations of Quantized Gauge Theories", in Current Algebra and Anomalies, S. B. Treiman et.al. Eds., World Scientific, Singapore, 1985

[8] See, for example, M. A. Nowak, M. Rho, and I. Zahed, Chiral Nuclear Dynamics, World Scientific, Singapore, 1996.

[9] R. K. Bhaduri, Models of the Nucleon: from Quarks to Soliton - (Lecture notes and supplements in physics), Addison-Wesley, Reading MA, 1988.

[10] I. Zahed and G. E. Brown, Phys. Rep. 142, 1 (1986).

[11] M. Creutz, I. J. Muzinich, and T. N. Tudron, Phys. Rev. D19, 531 (1979).

[12] J. L. Gervais and B. Sakita, Phys. Rev. D18, 453 (1978).

[13] M. Belloni, An Operator Approach to Gauss' Law in Yang-Mills Theory and Quantum Chromodynamics. University of Connecticut Ph.D. dissertation, 1997; (unpublished) Appendix F.

[14] H. Keller, Numerical Solution of Two Point Boundary Value Problems, SIAM, Philadelphia, 1976.

[15] E. Coddington and N. Levinson, Theory of Ordinary Differential Equations, McGraw Hill, New York, 1955.

[16] W. Rudin, Principle of Mathematical Analysis, third edition, McGraw Hill, New York, 1976. 


\section{FIGURE CAPTIONS}

Figure 1. A solution of Eq.(A4)) is plotted against the dimensionless variable $t$. The input into the equation is given in Subsection IVA. In this case, $\ell$, the winding number of the gauge field $\mathcal{N}$, is given by $\ell=0$, and the integer that characterizes the $\lim _{t \rightarrow \infty} \overline{\mathcal{N}}$, is $m=7$. $Q=\frac{7}{2}$ for this case.

Figure 2. A solution of Eq. (A4) is plotted against the dimensionless variable $t$. The input into the equation is given in Subsection IVB. In this case, $\ell$, the winding number of the gauge field $\mathcal{N}$, is given by $\ell=0$, and the integer that characterizes the $\lim _{t \rightarrow \infty} \overline{\mathcal{N}}$, is $m=5$. $Q=\frac{5}{2}$ for this case.

Figure 3. A solution of Eq. A4) is plotted against the dimensionless variable $t$. The input into the equation is given in Subsection IVQ. In this case, $\ell$, the winding number of the gauge field $\mathcal{N}$, is given by $\ell=0$, and the integer that characterizes the $\lim _{t \rightarrow \infty} \overline{\mathcal{N}}$, is $m=9$. $Q=\frac{9}{2}$ for this case.

Figure 4. A solution of Eq. (A4) is plotted against the dimensionless variable $t$. The input into the equation is given in Subsection IVD. In this case, $\ell$, the winding number of the gauge field $\mathcal{N}$, is given by $\ell=1$, and the integer that characterizes the $\lim _{t \rightarrow \infty} \overline{\mathcal{N}}$, is $m=9$. $Q=5.5677$ for this case.

Figure 5. A solution of Eq. (A4) is plotted against the dimensionless variable $t$. The input into the equation is given in Subsection IVE. In this case, $\ell$, the winding number of the gauge field $\mathcal{N}$, is given by $\ell=1$, and the integer that characterizes the $\lim _{t \rightarrow \infty} \overline{\mathcal{N}}$, is $m=5$. $Q=3.5677$ for this case.

Figure 6. A solution of Eq. (A4) is plotted against the dimensionless variable $t$. The input into the equation is given in Subsection IVF. In this case, $\ell$, the winding number of the gauge field $\mathcal{N}$, is given by $\ell=1$, and the integer that characterizes the $\lim _{t \rightarrow \infty} \overline{\mathcal{N}}$, is $m=11$. $Q=6.5677$ for this case. 